\title{
Syntactic Model-Based Human Body 3D Reconstruction and Event Classification via Association based Features Mining and Deep Learning
}

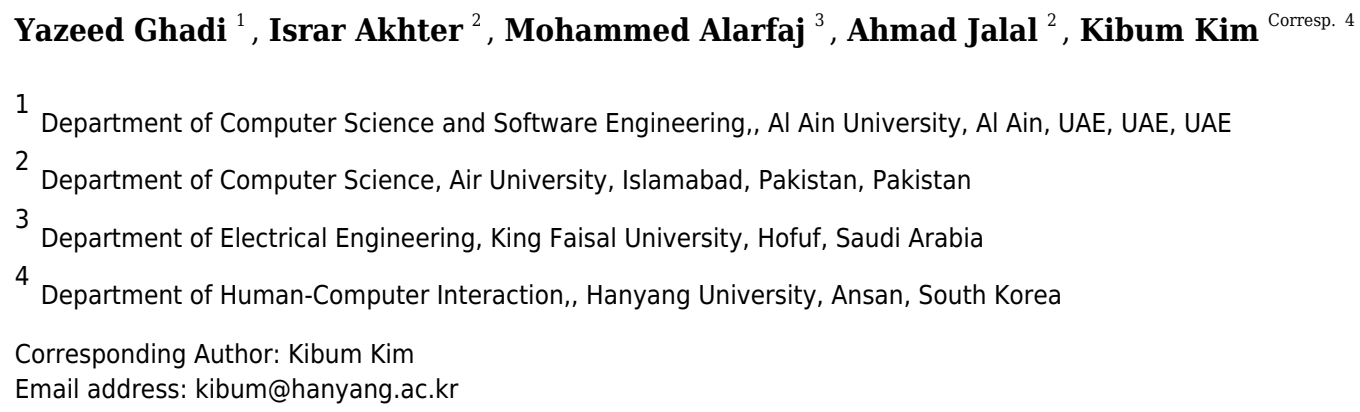

The study of human posture analysis and gait event detection from various types of inputs is a key contribution to the human life log. With the help of this research and technologies humans can save costs in terms of time and utility resources. In this paper we present a robust approach to human posture analysis and gait event detection from complex videobased data. For this, initially posture information, landmark information are extracted, and human 2D skeleton mesh are extracted, using this information set we reconstruct the human 2D to 3D model. Contextual features, namely, degrees of freedom over detected body parts, joint angle information, periodic and non-periodic motion, and human motion direction flow, are extracted. For features mining, we applied the rule-based features mining technique and, for gait event detection and classification, the deep learning-based CNN technique is applied over the mpii-video pose, the COCO, and the pose track datasets. For the mpii-video pose dataset, we achieved a human landmark detection mean accuracy of $87.09 \%$ and a gait event recognition mean accuracy of $90.90 \%$. For the COCO dataset, we achieved a human landmark detection mean accuracy of $87.36 \%$ and a gait event recognition mean accuracy of $89.09 \%$. For the pose track dataset, we achieved a human landmark detection mean accuracy of $87.72 \%$ and a gait event recognition mean accuracy of $88.18 \%$. The proposed system performance shows a significant improvement compared to existing state-of-the-art frameworks 


\title{
1 Syntactic Model-Based Human Body 3D
}

2 Reconstruction and Event Classification via

\section{${ }_{4}$ Association bas Learning}

5

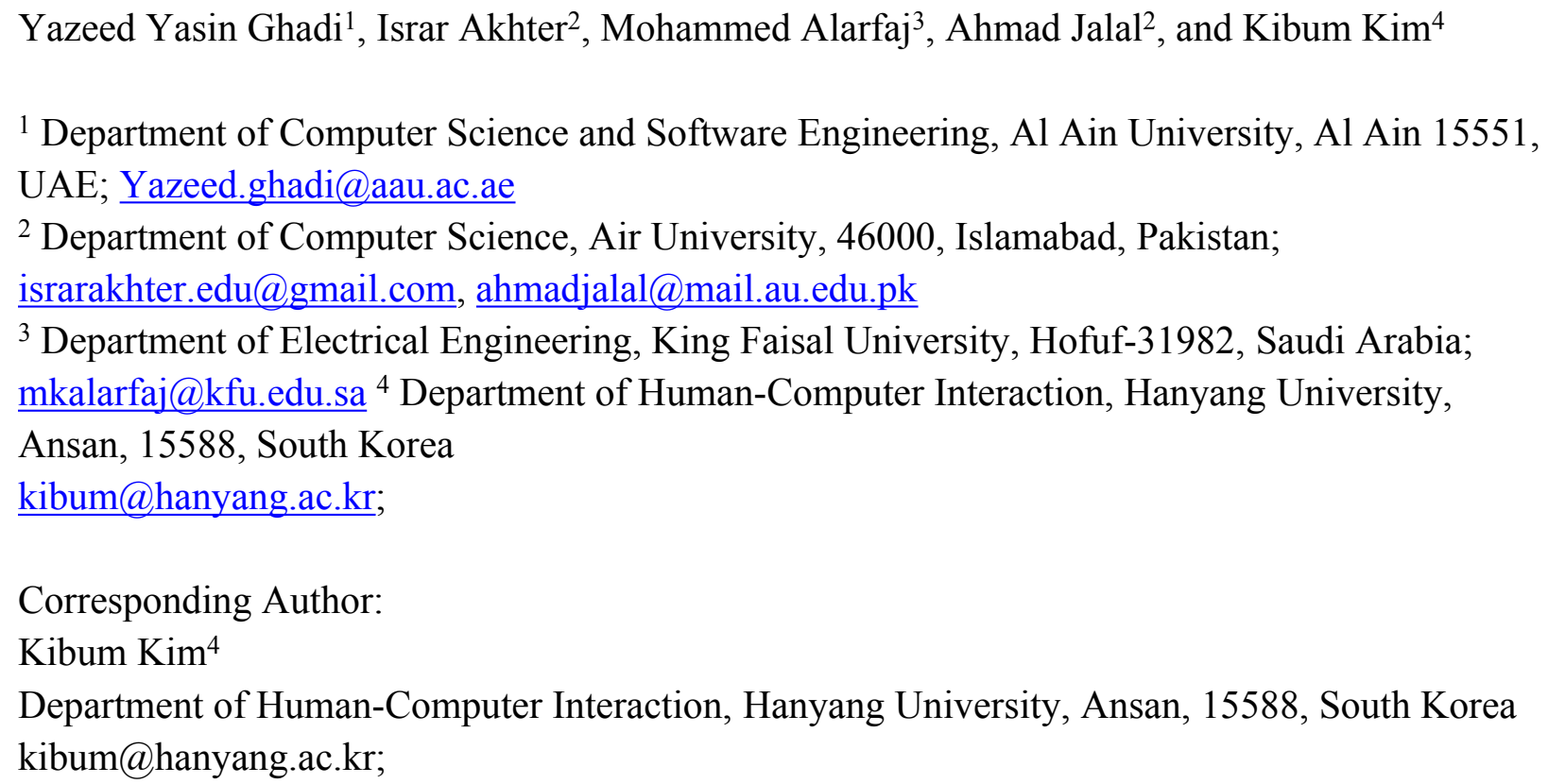

\begin{abstract}
The study of human posture analysis and gait event detection from various types of inputs is a key contribution to the human life log. With the help of this research and technologies humans can save costs in terms of time and utility resources. In this paper we present a robust approach to human posture analysis and gait event detection from complex video-based data. For this, initial posture information, landmark information are extracted, and human 2D skeleton mesh are extracted, using this information set we reconstruct the human 2D to 3D model. Contextual features, namely, degrees of freedom over detected body parts, joint angle information, periodic and non-periodic motion, and human motion direction flow, are extracted. For features mining, we applied the rule-based features mining technique and, for gait event detection and classification, the deep learning-based CNN technique is applied over the mpii-video pose, the COCO, and the pose track datasets. For the mpii-video pose dataset, we achieved a human landmark detection mean accuracy of $87.09 \%$ and a gait event recognition mean accuracy of $90.90 \%$. For the $\mathrm{COCO}$ dataset, we achieved a human landmark detection mean
\end{abstract}


39

40

41

42

43

44

45

46

47

48

49

50

51

52

53

54

55

56

57

58

59

60

61

62

63

64

65

66

67

68

69

70

71

72

73

74

75

80 accuracy of $87.36 \%$ and a gait event recognition mean accuracy of $89.09 \%$. For the pose track dataset, we achieved a mean accuracy of $87.09 \%$ in human landmark detection and a gait event recognition mean accuracy of $88.18 \%$. The proposed system performance shows a significant improvement compared to existing state-of-the-art frameworks.

Subjects Artificial Intelligence, Computer Vision, 2D/3D reconstruction, Deep

learning, Machine learning

Keywords 2D to 3D Reconstruction, Convolutional Neural Network, Gait Event

Classification, Human Posture Analysis, Landmark Detection, Synthetic Model, Silhouette Optimization.

\section{INTRODUCTION}

With regard to human posture information, motion estimation, and gait event detection from various types of input such as camera-based data, sensor-based datasets currently provide the most challenging issues. Various approaches and models are proposed to find more accurate and appropriate methods and functions for event classification and human body posture, motion and movement analysis. Data generation, communication, and transmission are routine takes for smart systems such as hospital management systems, educational systems, emergency systems, communication systems $u d$ din Tahir, Jalal \& Kim; ud din Tahir (2020), store records, airport or other transportation systems Jalal, Khalid \& Kim, 2020; Khalid et al (2021). The generated data is needed to be processed and utilized in the context of finding some useful information for humans in terms of time-saving, and reducing the cost of manpower Jalal, Khalid \& Kim (2020). Human posture and gait event analysis can help us represent human movement as information that is useful in smart systems for the detection and identification of human events and conditions such as standing, walking, running, playing, singing and dancing.

Various smart systems such as smart surveillance systems, cryptography in smart systems, and data security systems ur Rehman, Raza \& Akhter (2018), management systems and smart sports systems save us time and manpower and thus also money. With smart systems, many aspects of the behavior and condition of patients can be monitored automatically and more efficiently, thus relieving the burden on medical staff and patient care and freeing them from some redundancies. For sports, we can recognize the current event such as classification about games. Smart security systems can detect the security status, security issues, normal and abnormal events in places such as airports, or defense facilities.

Following are the main key points of problem statements.

- In various crowd-based systems, human motion analysis is a challenge to perform and archive.

- For human motion analysis, mostly 2D human model was suggested and, in this regard, 3D reconstruction is proposed. 
81

82

83

84

85

86

87
- For data optimization, most of the research was based on traditional data optimization techniques. To deal with this, data mining techniques are proposed.

- Event recognition is challenging in the computer vision domain, especially when complex human crowd-based data is used as an input of the system. To tackle this problem, we adopted contextual features and $\mathrm{CNN}$-based classification.

\section{Technical Gaps:}

In this domain, the various model was proposed with various techniques. Many papers deal with human motion analysis and gait event detection separately. In the above papers, research gaps and technical gaps are related throughout input-output. Initially, input is based on video-based data, and pre-pressing is performed to deal with data processing cast and time-saving. After that, the motion analysis of human detection, human landmark, human 2D stick model, and 3D human reconstruction are performed. This technique is robust to find and analyze the human motion information data which was normally dealt with in previous papers and was based on the gap of human motion methods. For event recognition, the pre-step to find the features with a data mining approach using CNN gait event detection and recognition is performed. On the other hand, previous papers deal with various models and data optimization which was the issue of processing cast and time.

\section{Contribution of this Paper:}

Thus, in this research article, we propose a robust method for human body posture analysis and human gait event detection. For this, we use the mpiivideo-pose dataset; the COCO dataset and the pose track dataset as input, and initially preprocess the video samples for frame conversion, motion blur noise reduction and resizing. The next step in human detection is to process these data sources by various algorithms for silhouette extraction, optimization of detected human silhouettes and human body landmark detection. Then, the next stage is to analyze the human posture information. For this, given 2D images to approximate 3D image reconstruction is applied using ellipsoid and synthetic modeling of the human body. This is followed by the feature extraction phase in which contextual features information is extracted. This information includes the degree of freedom, periodic motion, non-periodic motion, motion direction, flow, and rotational and angular joint features. For features mining, an association-based technique is adopted. For gait event classification, CNN is applied.

The main contribution of this paper is:

- With complex datasets, the detection of and optimization of humans and the extraction of and optimization of human silhouettes are challenging. We, therefore, propose a robust human silhouette extraction approach.

- For human motion, posture, and movement information analysis, we propose a method for the conversion of the human skeleton-based 2D mesh to the 3D human skeleton. 
126

127

128

129

130

131

132

133

134

135

136

137

138

139

140

141

142

143

144

145

146

147

148

149

150

151

152

153

154

155

156

157

158

159

160

162

163

164

165

166

167

168

169

170

171

172
- For the detection and recognition of gait events and contextual features, the extraction approaches are proposed in which degrees of freedom (DOF), periodic motion, non-periodic motion, motion direction, flow, and rotational angular joint features are extracted.

- Finally, data mining and classification via hierarchical methods, mining, and CNN-based methods are adopted for gait event classification.

The subdivisions of this article are as follows: we start with related works, followed by our system methodology, then, the detailed experimental setup discussion and, finally, an overview of the paper is presented in the conclusion.

\section{RELATED WORK}

Innovations in smartphone cameras and recorded video and developments in object marker sensor-based devices allow for more efficient farming and collection of data for exploration and research in the area. Several novel and effective approaches for recognizing human events, movements, and postures have been developed in the past. Table 1 includes a comprehensive review of recent research in this area.

\section{MATERIALS \& METHODS}

For the video input of our proposed method, the main source is RGB cameras which provide clean and noise-free data. The first step is video to frame conversion $30 \mathrm{fps}$ which reduces computational cost and time. After this, noise reduction techniques are applied. Human detection is achieved using Markov random field, change detection, floor detection, and spatial-temporal dereferencing. Then, 3D human reconstruction is achieved in which computational models with ellipsoids, the synthetic model with supper quadrics, joint angle estimation, and 3D reconstruction are applied. After this contextual features extraction is applied. For features mining we applied association-based techniques. Finally, gait event classification is achieved with the help of CNN over three state-of-the-art datasets. Figure 1 demonstrates the proposed system model's structural design.

Figure 1. The proposed system model's structural design.

The complete overview of the proposed method is described in (Algorithm 1) in which steps of the proposed method are described and main functions with sub-functions and equations are mentions.

The graphical superstations show the flow of every program, system, or software. Figure 2 shows the graphical repetition in the shape of a flow chart of the proposed methodology. 
Figure 2. Flow chart of the proposed method.

173

174

175

176

177

178

179

180

181

182

183

184

185

186

187

188

189

190

\section{Preprocessing of the data}

Before the detection of human body landmarks, some preprocessing methods are applied to save computational cost and time. Initially, video data is converted into images and then a motion blur filter is applied to reduce excess information.

\section{Background subtraction}

For background subtraction, we applied an optimized merging method technique in which we initially applied Markov random field based on color information and region merging methods. After this, change detection in image sequence is applied over an adaptive threshold-based approach, floor detection, and finally spatial-temporal differencing is adopted to get more accurate results. Figure 2 shows the results of background subtraction techniques.

Figure 3. Results of different background subtraction techniques along with the original image. (a) Original image (b) change detection (c) floor detection (d) Markov random field and (e) spatial-temporal differencing.

\section{Silhouette optimization and human detection}

In this sub-phase of landmark detection, we find the optimized human silhouette through the merging of change detection, floor detection, Markov random field, and spatial-temporal differencing techniques with the help of an adaptive threshold approach. (Algorithm 2) shows the detailed procedure of silhouette optimization.

After this, human detection is performed in two phases, initially, head detection is performed with the help of a human head "size and shape-based" technique. We set the weight of a human head as $\mathrm{w} 0=1 / 25$ of the human silhouette and, using region of interest model, we find the super pixel position of the human body and, after this, Gaussian kernel is used to capture the likely area of the human head. Finally, using this human head information, human shape and appearance information, human body movement, and motion information, human detection and identification is performed. Eq. (1) is used for head tracking

$$
T_{H e}^{q} \leftarrow T_{H e}^{q-1}+\Delta T_{H e}^{q-1}
$$

where $T_{H e}^{q}$ represents a human head land-mark location in any given video frame $q$ which is consequential to calculating by the frame differences. For human detection, Eq. (2) shows the mathematical relationship. 
215

216

217

218

219

220

221

222

223

224

225

226

227

228

229

230

231

232

233

234

235

236

237

238

239

240

241

242

243

244

245

246

247

248

249

250

251

252

253

254

255

256

257

$$
T_{F H}^{q}=\left(T_{H e}^{q} \leftarrow T_{H e}^{q-1}+\Delta T_{H e}^{q-1}\right)+T_{E n d}^{q}
$$

where $T_{F H}^{q}$ represents a human location in any given video frame $q$ and $T_{\text {End }}^{q}$ shows the bounding box size for human detection. Figure 3 shows the results of optimized human body silhouettes, head detection, and human detection.

(a)

(b)

(c)

Figure 4. Results of (a) Optimized human silhouette (b) human head detection (c) human detection in RGB videos and image sequences.

Once human silhouette extraction and human detection are achieved, the next phase is to find human body landmarks for the posture estimation and analysis of the human body movements.

\section{Body landmarks detection}

In this sub-phase of landmark detection, we establish human body landmarks using a fast marching algorithm; we have applied this to the full human silhouette. Initially, the center point of the human body is extracted for the distance value $\operatorname{dis}(h)=0$, where $h$ is the initial point and is distinguished as a marked point. All remaining unmarked points of the human body are considered as $\operatorname{dis}(p)=\infty$. this process is applied to every detected point and to the pixel value of the human silhouette. The mathematical representation is:

$$
\begin{gathered}
\text { dis }=\left\{\frac{d i s_{x}+d i s_{y}+\sqrt{\Delta}}{2}\right\} \text { when } \Delta \geq 0 \\
\min \left(d i s_{x}+d i s_{y}\right)+\text { wi otherwise } \\
\Delta=x^{2}-\left(d i s_{x}-d i s_{y}\right)^{2}
\end{gathered}
$$

where $d i s_{x}$ and $d i s_{y}$ is the geodesic distance in the $2 \mathrm{~d}$ plane, correspondingly, $\quad d i s_{y}=\min (\operatorname{Disi}+1, m o, D i s i-1, m o)$ and $d i s_{y}$ $=\min ($ Disi,no $+1, D i, n o-1)$. After this, human body parts estimation is performed by finding the midpoint of the human body and the hands, elbows, neck, head, knees, and feet points are extracted Gochoo et al. (2021). The detection of the human midpoint is represented as;

$$
T_{t}^{q} \leftarrow T^{q-1}+\Delta T_{t o}^{q-1}
$$

where $\mathrm{T}_{\mathrm{t}}^{\mathrm{q}}$ represents a human midpoint location in any given video frame q which is consequential to calculation according to frame differences Akhter, Jalal \& Kim, (2021). To find the knees points we take the midpoint between the human-body midpoint and the two feet points. Eq. (6) demonstrates the human knee points;

$$
T_{k}^{q}=\left(T_{t}^{q}-T_{f}^{q}\right) / 2
$$

where $T_{k}^{q}$ is a knee point, $T_{m}^{q}$ is the human body midpoint, and $T_{f}^{q}$ denotes a foot point. For each elbow position estimation we utilized the neck point and respective (left/right) hand point information and found the mid point between the hand and the neck points. Eq. (7) as; 
258

259

260

261

262

263

264

265

266

267

268

269

270

271

272

273

274

275

276

277

278

279

280

Figure 5. Human body landmark detection results (a) presents the landmark results using an HSV color map, (b) presents the eleven human body points.

where $T_{e}^{q}$ denotes the human elbow point, $T_{h n}^{q}$ is the human hand point, and $T_{n q}^{q}$ denotes the neck point. Figure 5 represents the results of landmarks and body parts.

$$
T_{e}^{q}=\left(T_{h n}^{q}-T_{n q}^{q}\right) / 2
$$

(1)

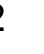


297

298

299

300

301 where $C_{m e}$ is the computational model with ellipsoids, $P_{a}\left(e_{x}, e_{y} e_{z,}\right)$ is the first point with the value of $x, y$ and $P_{a+1}\left(e_{x}, e_{y}\right)$ is the next point of the human body with the value of $x, y$. Figure 7 shows the results of the computational model with ellipsoids over human body points.

Figure 7. The results of the computational model with ellipsoids over human body points.

After completion of the computational model with ellipsoids, the next phase is the synthetic model with super quadrics.

\section{B. Synthetic model with super quadrics}

To display the human posture and estimation of human motion the synthetic model with super quadrics is adopted, using the computational model with ellipsoids information we utilize the previous ellipsoids and convert them into a rectangular shape for more accurate information and analysis of human posture.

$$
S_{S Q}=C_{m e} \rightarrow S_{e}
$$

Where $S_{S Q}$ is the synthetic model with super quadrics and $C_{m e}$ is the computational model with ellipsoid information, $\rightarrow s_{e}$ is the reshaping of the given information of points. Figure 8 shows the results of the synthetic model with super quadrics over the computational model with ellipsoids.

(a)

(b)

(c)

Figure 8. The results of the synthetic model with super quadrics over human body points. (a) Human 2D skeleton, (b) computational model with ellipsoids (c) synthetic model with super quadrics.

C. Joint angle estimation

For $3 \mathrm{D}$ reconstruction of the ellipsoids, the prerequisite step is to estimate joint angle information. For this, volumetric data and the edge information of the human body key points are extracted. For movement and angle information using the global and local coordinate system, we estimate the DOF for human body key points root information. After that, the Cartesian product of the skeleton graph is estimated for further processing.

$$
T_{N e}^{q} \leftarrow T_{N e}^{q-1}+\Delta T_{N e}^{q-1}
$$

where $T_{N e}^{q}$ represents the neck landmark location in any given video frame, $q$ is consequential to calculation of the frame differences. See Figure. 9. 
Figure 9. The theme concept of local and global coordinate systems. The left side shows the local coordinate system over the human left knee; the right side shows the DOF based global coordinate system.

331

332

333

334

335

336

337

338

339

340

341

342

343

344

345

346

347

348

349

350

351

352

353

354

355

356

357

358

359

360

361

362

363

364

365

366

367
$F_{v}=\left[\theta_{g_{-}}, \theta_{S_{-} h}, \theta_{S_{-},}, \theta_{R_{-} e}, \theta_{L_{-} e}, \theta_{R_{-} h}, \theta_{L_{-}}, \theta_{S_{-} m^{\prime}}, \theta_{R_{-},}, \theta_{L_{-} k}, \theta_{R_{-} f}, \theta_{L_{-} f},\right]$

where $F_{v}$ represents the angle joint function, $\theta_{g_{-} l}$ denotes the global to local coordinates, $\theta_{S_{-} h}$ indicates the head point, $\theta_{S_{-} n}$ shows the neck point, $\theta_{R_{-} e}$ denotes the right elbow point, $\theta_{L_{-} e}$ indicates the left elbow point, $\theta_{R_{-} h}$ represents the right-hand point, $\theta_{L_{-} h}$ shows the left-hand point, $\theta_{S_{-} m}$ indicates the mid-point, $\theta_{R_{-} k}$ shows the right knee point, $\theta_{L_{-} k}$ denotes the left knee point, $\theta_{R_{-} f}$ indicates the right foot point, $\theta_{L_{-} f}$ shows the left foot point.

\section{D. $3 D$ ellipsoid reconstructions}

Finally, the 3D reconstruction of the human body is implemented using human body joint information, ellipsoid information, and skeleton graphs. The preview of the 3D image gives us more precise and accurate posture information and estimation for further processing.

$$
R_{E}(x, 0 \mid I, D) \propto R_{E}(x) R_{E}(I \mid x) R_{E}(D \mid x) R_{E}(D \mid \theta)
$$

Where $R_{E}(x, 0 \mid I, D)$ is the $3 \mathrm{D}$ reconstruction ellipsoid, $\propto$ is the reshaping and $R_{E}(x) R_{E}(I \mid x) R_{E}(D \mid x) R_{E}(D \mid \theta)$ shows the angle information based on previous data. Figure 10 shows the results of the $3 \mathrm{D}$ ellipsoid reconstruction over the synthetic model with super quadrics and joint angle estimation.

Figure 10. The results of the 3D ellipsoid reconstruction over the synthetic model with super quadrics and joint angle estimation.

\section{Contextual features extraction}

In this section, the extraction of contextual features is implemented in which DOF, periodic motion, non-periodic motion, motion direction and flow and rotational angular joint features are extracted.

\section{A. Degree of freedom}

In contextual features extraction, Degree of freedom (DOF) is implemented over all body parts and the $\mathrm{x}, \mathrm{y}, \mathrm{z}$ dimension information In DOF features vector three directional angle values for each body parts, for knee points x_knee, y_knee, z_knee, for head points $x \_h e a d, y \_h e a d, z \_h e a d$, for neck points $x_{-}$neck, $y_{-}$neck, $z_{-}$neck, for elbow points $x_{-}$elbow, $y_{-}$elbow, $z_{-}$ elbow, for hand points $\mathrm{x}_{-}$hand, $\mathrm{y}_{-}$hand, $\mathrm{z}_{-}$hand, for midpoints $\mathrm{x}_{-}$mid, $\mathrm{y}_{-}$ mid, $z_{-}$mid, for foot points $x_{-}$foot, $y_{-}$foot, $z_{-}$foot. Eq. (16) shows the mathematical relation for DOF. 
368

369

370

371

372

373

374

375

376

377

378

379

380

381

382

383

384

385

386

387

388

389

390

391

392

393

394

395

396

397

398

399

400

401

402

403

404

405

$$
D_{o f}=A(\theta x, \theta y, \theta z) \uparrow \varepsilon D
$$

where $D_{o f}$ represents the degree of freedom feature vector, $\theta x, \theta y, \theta z$ shows the three dimension of the angle and $\varepsilon D$ is the local and global coordinate system. Figure 11 shows the results for the degree of freedom:

Figure 11. Few DOF results examples.

\section{B. Periodic motion}

In this contextual feature, human motion is detected over human body parts. The targeted area of interest is the human body portion which provides periodic motion. The detection of this area is performed with the base analysis of the human body. A bounding box indicates the region of interest. The Eq. (17) shows the mathematical relation for periodic motion.

$$
P M(t)=\alpha \sin (\omega t+k)
$$

where $P M(t)$ denotes periodic motion and $\alpha \sin (\omega t+k)$ shows the relation of human motion that is repeated in any given sequence of images. Figure 12 shows the results of periodic motion:

\section{Figure 12. Periodic motion results.}

\section{Non-periodic motion}

In the non-periodic motion contextual feature, human motion is detected over human body parts. The targeted area of interest is the human body portion which provides nonperiodic motion and non-uniform motion. The detection of this area is performed with the base analysis of human body motion. A bounding box indicates the region of interest. Eq. (18) shows the mathematical relation of non-periodic motion:

$$
\operatorname{NPm}(t)=\left\|P_{t, t+1}-P_{t, t+2}\right\|
$$

where $N P m(t)$ denotes non-periodic motion and $P_{t, t+1}-P_{t, t+2}$ shows the difference between the first and the next sequence of images in input data. Figure 13 shows the results of non-periodic motion:

Figure 13. Results of non-periodic motion.

\section{Motion direction flow}

For the identification of more accurate gait events motion direction flow is one a contributions in terms of contextual features. Using changes in motion and human motion body flow we detect the direction of human body 
406

407

408

409

410

411

412

413

414

415

416

417

418

419

420

421

422

423

424

425

426

427

428

429

430

431

432

433

434

435

436

437

438

439

440

441

442

443

444

445 movement and motion flow. Eq. (19) shows the mathematical model for motion direction flow features.

$$
M_{d f}=\sum_{0}^{p} I_{v l}(I) \rightarrow D
$$

where $M_{d f}$ is motion direction flow of the human body, $I$ is the index values of the given image, $I_{v l}$ is RGB $(\mathrm{x}, \mathrm{y}, \mathrm{z})$ pixel indexes, and $\rightarrow D$ shows the motion direction. Figure 14 shows the results of motion direction flow over the basketball class video.

Figure 14. The results of motion direction flow over the basketball video.

\section{E. Rotational angular joint}

Rotational angular joint features are based on the angular geometry of human body parts. A 5 X 5 pixel region is used over detected body parts and from every node of the window of pixel region $\cos \theta$ is estimated and maps all values in the feature vector. The Eq. (20) shows the mathematical model of rotational angular joint features.

$$
\begin{aligned}
& A 1=\cos (x, y) \rightarrow L, A 2=\cos (x, y) \rightarrow L \\
A 3= & \cos (x, y) \rightarrow L, A 4=\cos (x, y) \rightarrow L
\end{aligned}
$$

where $A 1, A 2, A 3, A 4$ denotes the sides of the $5 \times 5$ windows, $\cos (x, y)$ represents the angle value over pixel $x$ and $y$, and $\rightarrow L$ indicates the side to follow. Figure 15 shows the results of rotational angular joint features over the dance class.

Figure 15. Rotational angular joint results and the pattern of rotational angels.

After the completion of the contextual features portion, we concatenate all the sub-feature vectors into the main feature vector while (Algorithm 3) shows the detailed overview of the contextual features extraction approach.

\section{Data optimization and features mining}

The association rule-based features mining method helps us to pick the most unique features that screen out unnecessary and inconsistent features from the extracted dataset which tend to reduce gait event classification precision and accuracy. This is a bottom-up strategy that starts with a null feature set $n f$ and progressively adds innovative features based on optimization function selection. This can decrease the mean square error which results in more significant details. The association rule-based features mining approach is 
446

447

448

449

450

451

452

453

454

455

456

457

458

459

460

461

462

463

464

465

466

467

468

469

470

471

472

commonly used in various domains such as security systems, medical systems and image processing-based smart systems.

This technique helps to minimize the main features space data while the features mining approach is dependent upon the specific objective function for an optimal solution which plays the key role in gait event classification. The Bhattacharyya distance calculation features optimization approach is used in the present architecture for various human event-based classes. It can determine the differentiation rating $n f_{(x, y)}$ among different segments $x$ and $b$ and then test it.

$$
n f_{(x, y)}=\left(g_{x}-g_{y}\right)\left(\frac{\Sigma_{x}-\Sigma_{y}}{2}\right)\left(g_{x}-g_{y}\right)^{t}
$$

where $n f_{(x, y)}$ is the optimal features set, $g_{x}$ are the mean and $\Sigma_{x}$ are the covariance of class $x$ and $g_{y}$ are the mean and $\Sigma_{y}$ are the covariance of class $y$ for $M$ numbers of event-based classes. The optimal solution score is computed as.

$$
O F v=\frac{1}{N^{2}} \sum_{u=1}^{M} \sum_{v=1}^{M} n f_{(u, v)}
$$

A recognition assessment criterion is suggested for estimating different gait event classifications for an input dataset to acquire selected features that can be expected to eliminate classification errors as well as provide improved inter-class interpretability throughout features data. For the mpii-video-pose dataset DOF, Periodic motion, Rotational angular joint features are selected. For the Pose track dataset, DOF, Motion direction and flow nonPeriodic motion features are selected. For the COCO dataset, Motion direction and flow nonPeriodic motion, Rotational angular joints features are selected. Figure 16 shows the most accurate features results over the mpii-video pose, the $\mathrm{COCO}$, and the Pose track datasets.

Figure 16. The most accurate features results via the rule-based features mining approach over the mpii-video pose, $\mathrm{COCO}$, and Pose track datasets.

\section{Event classification}

475

476

477

478

479

480

481

482

483

484

485

486

The extracted optimal features vector is used as input for Convolution Neural Network (CNN) for gait event classification. CNN is a deep learning-based classification approach which is widely used in image and video types of input data. CNN works well and it gives more accurate results compared to other traditional techniques. $\mathrm{CNN}$ adds less processing weight with minimum bias, thus providing a high accuracy rate.

The input, output, and hidden layers are the three main layers in a Convolutional Neural Network (CNN). The convolutional layer, important mechanisms, complete linked layer, and standardization layer are the four sub-divisions of each secret layer. The sub-band extracted features are across in the input neurons and highly correlated throughout the convolutional layer by a $5 x 5$ graded selector. The batch normalization method is then used to aggregate the responses among all neuronal populations. To further minimize feature dimensions, clustered solutions are convolutional and combined again, and then the interaction maps are computed by the completely connected sheet. 
491

492

493

494

495

496

497

498

499

500

501

502

503

504

505

506

507

508

509

510

511

512

513

514

515

516

517

518

519

520

521

522

523

524

525

526

527

$$
T R_{p}=\sum_{q} w i_{p . q} \times a_{p}+b_{q}
$$

where $T R_{p}$ is the CNN transfer function, $w i_{p . q}$ is the connecting layer's adjacent weight, $a_{p}$ shows the input optimized features vectors and $b_{q}$ is the bias values. Furthermore, the regression algorithm is adopted for parameter optimization and it reduces backpropagation errors. The output of the regression algorithm $\sigma\left(T R_{p}\right)$ returns the distribution function of total probability for possible $n$ repetition over the output layer of CNN.

$$
\sigma\left(T R_{p}\right)=\frac{e^{T R_{p}}}{\sum_{n=1}^{n} e^{T R_{p}}}, q=1, \ldots \ldots \ldots . . . n
$$

Figure 17 shows the detailed process of CNN parameters learning over gait event detection and classification:

Figure 17. CNN model overview.

\section{RESULTS}

\section{Dataset Descriptions}

The Mpii-video pose data set is a large-scale dataset, which contains human activities and posture information-based videos. 21 different activities such as home activities, lawn, garden, sports, washing windows, picking fruit, and rock climbing. All the videos All the videos selected for our dataset collection have been recommended as YouTube top 10 videos in each activity. Figure 18 shows some example images of the Mpii-video-pose dataset.

Figure 18. A few example images of the mpii-video-pose dataset.

The COCO (Common Objects in Context) dataset is based on multi-person tracking and object detection dataset, different activities contains in the COCO dataset They include Bicycling, Conditioning exercise, Dancing, Fishing and hunting, Music playing, Religious activities, Sports, Transportation, Walking, Water activities, winter activities. Figure 19 shows some example images of the COCO dataset:

\section{Figure 19. A few example images from the COCO dataset.}

Pose track dataset is based on two main tasks including multi-person human pose estimation and analysis over a single frame. Videos and articulated tracking have been based on human posture estimation. Dataset mostly consists of complex videos such as crowded or team sports. Various activities 
528

529

530

531

532

533

534

535

536

537

538

539

540

541

542

543

544

545

546

547

548

549

550

551

552

553

554

555

556

557

558

559

560

561

562

563

564

565

566

567

568 have been covered in the pose track dataset. Figure 20 shows some example images of the pose track dataset.

Figure 20. A few example images of the postmark dataset.

\section{Experiment I: The Landmarks Detection Accuracies}

To calculate the effectiveness and precision of the detected body parts, we approximate the geodesic distance Akhter (2020) Akhter, Jalal \& Kim (2021) from the given ground truth (GT) of the input datasets by Eq. (25):

$$
\text { Die }=\sqrt{\sum_{n=1}^{M}\left(\frac{O_{n}}{P_{n}}-\frac{O_{n}}{P_{n}}\right)^{2}}
$$

Here, $O$ is the ground truth values of the datasets and $P$ is the current location of the recognized body part. The error margin of 16 is set to identify the accuracy among the acknowledged body part value and the input data. Through Eq. (26), the proportion of the recognized body parts encircled within the error margin value of the considered data is known as;

$$
D e=\frac{100}{n}\left[\sum_{n=1}^{i}\left\{\begin{array}{rrr}
1 & \text { if } \begin{array}{c}
D e \leq 16 \\
0
\end{array}
\end{array}\right]\right.
$$

In (Table 2), columns 2, 4, and 6 show the error distances from the given dataset ground truth and columns 3,5, and 7 show the body part recognition and detection accuracies over the MPII, COCO and Posetrack datasets respectively.

$\mathrm{HP}=$ Head point, $\mathrm{NP}=$ Neck point, $\mathrm{REP}=$ Right elbow point, $\mathrm{RHP}=$ Right hand point, $\mathrm{LEP}=$ Left elbow point, $\mathrm{LHP}=$ Left hand point, $\mathrm{MP}=$ Mid-point, $\mathrm{LKP}=$ left knee point, $\mathrm{RKP}=$ Right knee point, $\mathrm{LFP}=\mathrm{Left}$ foot point, $\mathrm{RFP}=$ Right foot point.

(Table 3 ) represents the results of multi-person human body parts for the mpiivideo-pose dataset. For identified body parts, we indicate with $\checkmark$ and for unidentified we adopted we use $x$. We attained a detection accuracy for human $1-63.63 \%$, human $2-72.72 \%$, human $3-63.63 \%$, human $4-72.72 \%$, human $5-72.72 \%$ and the mean detection accuracy of $69.09 \%$.

$\mathrm{HP}=$ Head point, $\mathrm{NP}=$ Neck point, $\mathrm{REP}=$ Right elbow point, $\mathrm{RHP}=\mathrm{Right}$ hand point, $\mathrm{LEP}=$ Left elbow point, $\mathrm{LHP}=$ Left hand point, $\mathrm{MP}=$ Mid-point, $\mathrm{LKP}=$ left knee point, $\mathrm{RKP}=$ Right knee point, $\mathrm{LFP}=\mathrm{Left}$ foot point, $\mathrm{RFP}=$ Right foot point.

(Table 4) represents the results of multi-person human body parts for the COCO dataset. For identified body parts, we indicated $\checkmark$ and for unidentified body parts we adopted $\boldsymbol{x}$. We a attained detection accuracy for human1 $81.81 \%$, human 2 - 72.72\%, human3 - 72.72\%, human4- 72.72\%, human5 $72.72 \%$, and the mean detection accuracy of $74.54 \%$. 
569

570

571

572

573

574

575

576

577

578

579

580

581

582

583

584

585

586

587

588

589

590

591

592

593

594

595

596

597

598

599

600

601

602

603

604

605

606

607

608

$\mathrm{HP}=$ Head point, $\mathrm{NP}=$ Neck point, $\mathrm{REP}=$ Right elbow point, $\mathrm{RHP}=$ Right hand point, $\mathrm{LEP}=$ Left elbow point, $\mathrm{LHP}=$ Left hand point, $\mathrm{MP}=$ Mid-point, $\mathrm{LKP}=$ left knee point, $\mathrm{RKP}=$ Right knee point, $\mathrm{LFP}=$ Left foot point, $\mathrm{RFP}=\mathrm{Right}$ foot point.

(Table 5) presents the results of multi-person human body parts for the pose track dataset. For identified body parts, we use $\checkmark$ and for unidentified we adopted use $x$. The detection accuracies follow: for human1- $63.63 \%$, human2 - $63.63 \%$, human $3-63.63 \%$, human $4-63.63 \%$, human $5-72.72 \%$ and the mean detection accuracy is $65.45 \%$.

$\mathrm{HP}=$ Head point, $\mathrm{NP}=$ Neck point, $\mathrm{REP}=$ Right elbow point, $\mathrm{RHP}=$ Right hand point, $\mathrm{LEP}=$ Left elbow point, $\mathrm{LHP}=$ Left hand point, $\mathrm{MP}=$ Mid-point, $\mathrm{LKP}=$ left knee point, $\mathrm{RKP}=$ Right knee point, $\mathrm{LFP}=$ Left foot point, $\mathrm{RFP}=\mathrm{Right}$ foot point.

\section{Experiment II: Event Classification Accuracies}

For gait event classification, we used a CNN-based deep learning approach. The design method is evaluated by the Leave One Subject Out (LOSO) crossvalidation method. In Figure. 21, the results over the mpii-video-pose dataset show $90.90 \%$ gait event classification and detection accuracy. After this, we applied the deep belief network over the Olympic sports dataset and found the stochastic remote sensing event classification results. Figure 22 represents the confusion matrix for the COCO dataset with $89.09 \%$ mean accuracy for gait event classification. Finally, CNN is applied over the pose track dataset, with the mean gait event classification accuracy of $88.18 \%$. Figure 23 represents the results in the shape of the confusion matrix for the pose track dataset.

Figure 21. Confusion matrix results using CNN over Mpii-video-pose dataset.

$\mathrm{BI}=$ Bicycling, $\mathrm{Ce}=$ Conditioning exercise, $\mathrm{Da}=$ Dancing, $\mathrm{Fh}=$ Fishing and hunting, $\mathrm{Mp}=$ Music playing, $\mathrm{Ra}=$ Religious activities, $\mathrm{SP}=$ Sports, $\mathrm{Tr}=$ Transportation, $\mathrm{Wi}=$ Walking, $\mathrm{Wa}=$ Water activities, $\mathrm{Wn}=$ Winter activities.

Figure 22. Confusion matrix results using CNN over the COCO dataset.

$\mathrm{BI}=$ Bicycling, $\mathrm{Da}=$ Dancing, $\mathrm{Ce}=$ Conditioning exercise, $\mathrm{Fh}=$ Fishing and hunting, $\mathrm{Ra}=$ Religious activities, $\mathrm{Mp}=$ Music playing, $\mathrm{SP}=$ Sports, $\mathrm{Wi}=$ Walking, $\mathrm{Tr}=$ Transportation, $\mathrm{Wa}=$ Water activities, $\mathrm{Wn}=$ Winter activities.

Peer] Comput. Sci. reviewing PDF | (CS-2021:04:60580:2:0:CHECK 8 Oct 2021) 
Figure 23. Confusion matrix results using CNN over the Pose track dataset

609

610

611

612

613

614

615

616

617

618

619

620

621

622

623

624

625

626

627

628

629

630

631

632

633

634

635

636

637

638

639

640

641

642

643

644

645

646

647

648

649

650

651

652

653
$\mathrm{Ce}=$ Conditioning exercise, $\mathrm{BI}=$ Bicycling, $\mathrm{Da}=$ Dancing, $\mathrm{Mp}=$ Music playing, $\mathrm{Fh}=$ Fishing and hunting, $\mathrm{Ra}=$ Religious activities, $\mathrm{Tr}=$ Transportation, SP $=$ Sports, $\mathrm{Wi}=$ Walking, $\mathrm{Wa}=$ Water activities, $\mathrm{Wn}=$ Winter activities.

\section{Experiment III: Comparison with Other Classification Algorithms}

In this segment, we equate the recall, precision, and $\mathrm{f}-1$ measure over the mpii-video-pose dataset, the COCO, and the posetrack dataset. For the classification of gait events we used Decision tree, Artificial Neural Network and we associated the consequences with the CNN. (Table 6) shows the results over the mpii-video-pose dataset, (Table 7) shows the results over the COCO dataset, and (Table 8) shows the results over the posetrack dataset.

$\mathrm{BI}=$ Bicycling, $\mathrm{Ce}=$ Conditioning exercise, $\mathrm{Da}=$ Dancing, $\mathrm{Fh}=$ Fishing and hunting, $\mathrm{Mp}=$ Music playing, $\mathrm{Ra}=$ Religious activities, $\mathrm{SP}=$ Sports, $\mathrm{Tr}=$ Transportation, $\mathrm{Wi}=$ Walking, $\mathrm{Wa}=$ Water activities, $\mathrm{Wn}=$ Winter activities.

$\mathrm{BI}=$ Bicycling, $\mathrm{Da}=$ Dancing, $\mathrm{Ce}=$ Conditioning exercise, $\mathrm{Fh}=$ Fishing and hunting, $\mathrm{Ra}=$ Religious activities, $\mathrm{Mp}=$ Music playing, $\mathrm{SP}=$ Sports, $\mathrm{Wi}=$ Walking, $\mathrm{Tr}=$ Transportation, $\mathrm{Wa}=$ Water activities, $\mathrm{Wn}=$ Winter activities.

$\mathrm{Ce}=$ Conditioning exercise, $\mathrm{BI}=$ Bicycling, $\mathrm{Da}=$ Dancing, $\mathrm{Mp}=$ Music playing, $\mathrm{Fh}=$ Fishing and hunting, $\mathrm{Ra}=$ Religious activities, $\mathrm{Tr}=\mathrm{Transportation}, \mathrm{SP}$ $=$ Sports, $\mathrm{Wi}=$ Walking, $\mathrm{Wa}=$ Water activities, $\mathrm{Wn}=$ Winter activities.

Experiment IV: Performance analysis and comprehensive analysis of features selection

For gait event classification, contextual features were proposed in this article. Four features are based upon the full human body that are DOF, periodic motion, non-periodic motion, and motion direction flow. One is based upon human body joints which are the rotational angular joints. Using these features we make a complete features vector to classify gait event detection with the help of data mining technique and CNN-based classification approach. To check the performance of our contextual features we adopted one more experiment in which various combinations of features are utilized to check the best combination. Initially, the combination of degree of freedom (DOF), periodic motion, and nonperiodic motion are used. After that, the combination of degree of freedom (DOF), periodic motion, nonperiodic motion, and motion direction flow, finally the combination of degree of freedom (DOF), periodic motion, nonperiodic motion, motion direction flow, and the rotational angular joint are used. The table 9 shows the overview of performance analysis. The complete combination shows more accurate results. 
654

655

656

657

658

659

660

661

662

663

664

665

666

667

668

669

670

671

672

673

674

675

676

677

678

679

680

681

682

683

684

685

686

687

688

689

690

691

692

693

694

695
$\mathrm{DOF}=$ degree of freedom, $\mathrm{PM}=$ periodic motion, $\mathrm{NM}=$ nonperiodic motion, $\mathrm{MDF}=$ motion direction flow, $\mathrm{RAJ}=$ rotational angular joint

Experiment V: Comparison of our Proposed System with State-ofthe-Art Techniques

Fan et al. (2015) developed a unique approach to estimate the human pose which is based on deep learning-based dual-source CNN. As the input they used patches of a given image and human body patches. After that they combined both contextual and local index values to estimate human posture with a better accuracy rate. Pishchulin et al. (2016) proposed a robust formulation as a challenge of subsection partitioning and labeling (SPLP). The SPLP structure, unlike previous two-stage methods that separated the identification and pose estimation measures, suggests the number of persons, certain poses, spatial proximity, including component level occlusions all at the same time. In Wei et al. (2016), convolutional position devices have an edge infrastructure for solving formal classifications based on computer vision that does not require visual type reasoning. We demonstrated that by transmitting increasingly refined confusion beliefs among points, a sequential framework consisting of convolutional networks is incapable of effectively training a structural component for the position. Jin et al. (2019) proposed SpatialNet and TemporalNet combined to form a single pose prediction and monitoring conceptual model: Body part identification and part-level temporal classification are handled by SpatialNet, while the contextual classification of human events is handled by TemporalNet. Bao et al. (2020) suggest a hand gesture identification-by-tracking system that incorporates pose input into both the video human identification and human connection levels. A person's position prediction with pose descriptive statistics is used in the first level to reduce the impact of distracting and incomplete human identification in images. Umer et al. (2020) present a method for detecting people in video which depends on key feature connections. Rather than training the system to estimate key-point communications on video sequences, the system is equipped to estimate human pose utilizing personality on massive scale datasets. Sun et al. (2018) suggested a technique for features extracted in which they remove guided optical flow and use a CNN-based paradigm to identify and classify human events. Rachmadi, Uchimura \& Koutaki (2016) described a method for dealing with event recognition and prevention using CNN and NNA (Network in Network Architecture) frameworks, which are the foundation of modern CNN. CNN's streamlined infrastructure, median, average, and commodity features are used to define human activities. Zhu et al. (2019) provide a detailed method for identifying incidents in security video. Throughout the TRECVID-SED 2016 test, their method outperformed others by a substantial margin by combining path modeling with deep learning. 
696

697

698

699

700

701

702

703

704

705

706

707

708

709

710

711

712

713

714

715

716

717

718

719

720

721

722

723

724

725

726

727

728

729

730

731

732

733

734

735
(Table 9) shows the gait event mean accuracy comparison with the other methods over the MPII, COCO and Pose track datasets.

\section{SCOPE OF THE PAPER:}

This article is based on human motion analysis and gait event detectionbased approaches, which are parts of computer vision, image processing, data science, machine learning, deep learning, neural networks, and artificial intelligence. Especially we can deploy this research project over airport security, railways station, seaports, bus stations, metro stations, real-time smart system environment and other smart surveillance systems. With complex human-based crowed data which is the input of the proposed system, human motion analysis and gait event classification are the challenging tasks, with the help of various techniques, features, and classification method. We achieve this with a much better accuracy rate than previous methods. This system works fine in less crowed based datasets and also on complex video data systems, but sometimes accuracy may be compromised due to various factors such as distance, location, shadow, and other illusion-based factors. This system also works on the real-time smart system to analyze human motion and gait event detection.

\section{FAILURE CASES AND LIMITATIONS:}

This article is based on human motion analysis and gait event detectionbased approaches, which are parts of computer vision, image processing, data science, machine learning, deep learning, neural networks, and artificial intelligence. Especially we can deploy this research project over airport security, railways station, seaports, bus stations, metro stations, real-time smart system environment and other smart surveillance systems. With complex human-based crowed data which is the input of the proposed system, human motion analysis and gait event classification are the challenging tasks, with the help of various techniques, features, and classification method. We achieve this with a much better accuracy rate than previous methods. This system works fine in less crowed based datasets and also on complex video data systems, but sometimes accuracy may be compromised due to various factors such as distance, location, shadow, and other illusion-based factors. This system also works on the real-time smart system to analyze human motion and gait event detection. Figure 24 shows the detailed overview of failures and limitations of the proposed method in which we can see the overlapping issue and complex scenarios to find human and human landmarks.

Figure 24. Some examples of limitations and failure cases 
737

738

739

740

741

742

743

744

745

746

747

748

749

750

751

752

753

754

755

756

757

758

759

760

761

762

763

764

765

766

767

768

769

770

771

772

773

774

775

776

777

778

779
Openpose Viswakumar et al., (2019) is a CNN-based approach that is used for the human motion estimation and the gait analysis in various domains. We applied openpsoe CNN classification and detected the human body parts. The mean accuracy for the MPII dataset was $84.90 \%$, the one for COCO dataset was $86.00 \%$ and the one for Posetrack dataset was $85.18 \%$. Table 11 shows the complete results of openpose $\mathrm{CNN}$.

$\mathrm{HP}=$ Head point, $\mathrm{NP}=$ Neck point, $\mathrm{REP}=$ Right elbow point, $\mathrm{RHP}=$ Right hand point, $\mathrm{LEP}=$ Left elbow point, $\mathrm{LHP}=\mathrm{Left}$ hand point, $\mathrm{MP}=$ Mid-point, $\mathrm{LKP}=$ left knee point, $\mathrm{RKP}=$ Right knee point, $\mathrm{LFP}=$ Left foot point, $\mathrm{RFP}=$ Right foot point.

Table 12 shows the detailed comparison of the openpose CNN model with the proposed method. Results show the better accuracy of our proposed method than the accuracy of the openpose method.

Figure 25 shows the detailed results of openpose CNN over human sillhouttes.

Figure 25. Results of the openpose CNN based classification

\section{CONCLUSION}

This article is based on a reconstituted 3D synthetic model of the human body, gait event detection and classification over complex human video articulated datasets. Three benchmark datasets were selected for experiments: mpiivideo-pose, $\mathrm{COCO}$, and pose tracking datasets. Initially, human detection and landmark recognition are performed. After that, 2D human skeletons are transformed into 3D synthetic-based models for the analysis of human posture. For features reduction and optimization, a rule-based features mining technique is adopted and finally, a deep learning classification algorithm $\mathrm{CNN}$ is applied for gait event recognition and classification. For the mpiivideo pose dataset, we achieve the human landmark detection mean accuracy of $87.09 \%$ and gait event recognition mean accuracy of $90.90 \%$. For the COCO dataset, we achieve the human landmark detection mean accuracy of $87.36 \%$ and gait event recognition mean accuracy of $89.09 \%$. For the pose track dataset, we achieve the human landmark detection mean accuracy of $87.72 \%$ and gait event recognition mean accuracy of $88.18 \%$. The proposed system's performance shows a significant improvement compared to existing state-of-the-art frameworks. The limitation of the proposed framework is due to the complexity in the videos and group density which make it is difficult to achieve more accurate results.

\section{Funding}

This research was supported by the Basic Science Research Program through the National Research Foundation of Korea (NRF), funded by the Ministry of Education (No. 2018R1D1A1A02085645). Also, this work was supported by the Korea Medical Device Development Fund grant funded by the Korea 
780

781

782

783

784

785

786

787

788

789

790

791

792

793

794

795

796

797

798

799

800

801

802

803

804

805

806

807

808

809

810

811

812

813

814

815

816

817

818

819

820

821

government (the Ministry of Science and ICT, the Ministry of Trade, Industry and Energy, the Ministry of Health \& Welfare, the Ministry of Food and Drug Safety) (Project Number: 202012D05-02).

\section{Competing Interests}

The authors declare that they have no competing interests.

\section{Author Contributions}

- Yazeed Yasin Ghadi conceived and designed the experiments, authored or reviewed drafts of the paper, and approved the final draft.

- Israr Akhter examined the experimental parameters, performed logical testing, analyzed the data as well as computational work, prepared figures and/or tables, and approved the final draft.

- Mohammed Alarfaj analyzed the data, play vital role in major revision and approved the final draft.

- Ahmad Jalal conceived and designed the experiments, figures and tables, authored or reviewed drafts of the paper, and approved the final draft.

- Kibum Kim conceived and designed the experiments, authored or reviewed drafts of the paper, and approved the final draft.

\section{Data Availability}

The following information was supplied regarding data availability.

Code and data are available in the Supplemental Files.

\section{Supplemental Information}

Supplemental information for this article can be found online at

\section{REFERENCES}

Aggarwal JK, Cai Q. 1999. Human Motion Analysis: A Review. Computer Vision and Image Understanding. DOI: 10.1006/cviu.1998.0744.

Akhter I. 2020. Automated Posture Analysis of Gait Event Detection via a Hierarchical Optimization Algorithm and Pseudo 2D Stick-Model. Ph.D. Thesis, Air University, Islamabad, Pakistan, December 2020.

Akhter I, Jalal A, Kim K. Pose Estimation and Detection for Event Recognition using SenseAware Features and Adaboost Classifier.

Akhter I, Jalal A, Kim K. 2021. Adaptive Pose Estimation for Gait Event Detection Using Context-Aware Model and Hierarchical Optimization. Journal of Electrical Engineering $\mid \&$ Technology:1-9.

Amft O, Tröster G. 2008. Recognition of dietary activity events using on-body sensors. Artificial Intelligence in Medicine. DOI: 10.1016/j.artmed.2007.11.007.

Bao Q, Liu W, Cheng Y, Zhou B, Mei T. 2020. Pose-guided tracking-by-detection: Robust multi-person pose tracking. IEEE Transactions on Multimedia 23:161-175.

Cao Z, Simon T, Wei S-E, Sheikh Y. 2017. Realtime multi-person $2 \mathrm{~d}$ pose estimation using part affinity fields. In: Proceedings of the IEEE conference on computer vision and pattern 
822

823

824

825

826

827

828

829

830

831

832

833

834

835

836

837

838

839

840

841

842

843

844

845

846

847

848

849

850

851

852

853

854

855

856

857

858

859

860

861

862

863

864

865

866

867

recognition. 7291-7299.

Einfalt M, Dampeyrou C, Zecha D, Lienhart R. 2019. Frame-Level Event Detection in Athletics Videos with Pose-Based Convolutional Sequence Networks. In: Proceedings Proceedings of the 2nd International Workshop on Multimedia Content Analysis in Sports MMSports '19. New York, New York, USA: ACM Press, 42-50. DOI: $10.1145 / 3347318.3355525$.

Fan X, Zheng K, Lin Y, Wang S. 2015. Combining local appearance and holistic view: Dualsource deep neural networks for human pose estimation. In: Proceedings of the IEEE conference on computer vision and pattern recognition. 1347-1355.

Feng Q, Gao C, Wang L, Zhao Y, Song T, Li Q. 2020. Spatio-temporal fall event detection in complex scenes using attention guided LSTM. Pattern Recognition Letters. DOI: 10.1016/j.patrec.2018.08.031.

Franco A, Magnani A, Maio D. 2020. A multimodal approach for human activity recognition based on skeleton and RGB data. Pattern Recognition Letters. DOI: 10.1016/j.patrec.2020.01.010.

Franklin RJ, Mohana, Dabbagol V. 2020. Anomaly Detection in Videos for Video Surveillance Applications using Neural Networks. In: Proceedings of the 4th International Conference on Inventive Systems and Control, ICISC 2020. DOI: 10.1109/ICISC47916.2020.9171212.

Gochoo M, Akhter I, Jalal A, Kim K. 2021. Stochastic Remote Sensing Event Classification over Adaptive Posture Estimation via Multifused Data and Deep Belief Network. Remote Sensing 13. DOI: 10.3390/rs13050912.

Golestani N, Moghaddam M. 2020. Human activity recognition using magnetic inductionbased motion signals and deep recurrent neural networks. Nature Communications. DOI: 10.1038/s41467-020-15086-2.

Hassner T, Basri R. 2006. Example based 3D reconstruction from single 2D images. In: 2006 Conference on Computer Vision and Pattern Recognition Workshop (CVPRW'06). 15.

Hu Y, Jiang D, Yan S, Zhang L, others. 2004. Automatic 3D reconstruction for face recognition. In: Sixth IEEE International Conference on Automatic Face and Gesture Recognition, 2004. Proceedings. 843-848.

Jalal A, Akhtar I, Kim K. 2020. Human Posture Estimation and Sustainable Events Classification via Pseudo-2D Stick Model and K-ary Tree Hashing. Sustainability 12:9814.

Jalal A, Khalid N, Kim K. 2020. Automatic recognition of human interaction via hybrid descriptors and maximum entropy markov model using depth sensors. Entropy. DOI: 10.3390/E22080817.

Jiang YG, Dai Q, Mei T, Rui Y, Chang SF. 2015. Super Fast Event Recognition in Internet Videos. IEEE Transactions on Multimedia. DOI: 10.1109/TMM.2015.2436813.

Jin S, Liu W, Ouyang W, Qian C. 2019. Pose-guided tracking-by-detection: Robust multiperson pose tracking In: Proceedings of the IEEE/CVF Conference on Computer Vision and Pattern Recognition (CVPR).

Khalid N, Gochoo M, Jalal A, Kim K. 2021. Modeling Two-Person Segmentation and Locomotion for Stereoscopic Action Identification: A Sustainable Video Surveillance System. Sustainability 13:970.

Khan MA, Javed K, Khan SA, Saba T, Habib U, Khan JA, Abbasi AA. 2020. Human action recognition using fusion of multiview and deep features: an application to video 
868

869

870

871

872

873

874

875

876

877

878

879

880

881

882

883

884

885

886

887

888

889

890

891

892

893

894

895

896

897

898

899

900

901

902

903

904

905

906

907

908

909

910

911

912

913

surveillance. Multimedia Tools and Applications. DOI: 10.1007/s11042-020-08806-9.

Khan MH, Zöller M, Farid MS, Grzegorzek M. 2020. Marker-based movement analysis of human body parts in therapeutic procedure. Sensors (Switzerland). DOI: 10.3390/s20113312.

van der Kruk E, Reijne MM. 2018. Accuracy of human motion capture systems for sport applications; state-of-the-art review. European Journal of Sport Science. DOI: 10.1080/17461391.2018.1463397.

Li A, Miao Z, Cen Y, Zhang X-P, Zhang L, Chen S. 2020. Abnormal event detection in surveillance videos based on low-rank and compact coefficient dictionary learning. Pattern Recognition 108:107355.

Liu J, Luo J, Shah M. 2009. Recognizing realistic actions from videos' in the Wild. In: 2009 IEEE Computer Society Conference on Computer Vision and Pattern Recognition Workshops, CVPR Workshops 2009. DOI: 10.1109/CVPRW.2009.5206744.

Lohithashva BH, Aradhya VNM, Guru DS. 2020. Violent video event detection based on integrated LBP and GLCM texture features. Revue d'Intelligence Artificielle 34:179-187.

Mokhlespour Esfahani MI, Zobeiri O, Moshiri B, Narimani R, Mehravar M, Rashedi E, Parnianpour M. 2017. Trunk motion system (TMS) using printed body worn sensor (BWS) via data fusion approach. Sensors (Switzerland). DOI: 10.3390/s17010112.

Pishchulin L, Insafutdinov E, Tang S, Andres B, Andriluka M, Gehler P V, Schiele B. 2016. Deepcut: Joint subset partition and labeling for multi person pose estimation. In: Proceedings of the IEEE conference on computer vision and pattern recognition. 4929 4937.

Rachmadi RF, Uchimura K, Koutaki G. 2016. Combined convolutional neural network for event recognition. In: Proceedings of the Korea-Japan Joint Workshop on Frontiers of Computer Vision. 85-90.

Sun S, Kuang Z, Sheng L, Ouyang W, Zhang W. 2018. Optical Flow Guided Feature: A Fast and Robust Motion Representation for Video Action Recognition. In: Proceedings of the IEEE Computer Society Conference on Computer Vision and Pattern Recognition. DOI: 10.1109/CVPR.2018.00151.

Tahir SB. 2020. A Triaxial Inertial Devices for Stochastic Life-Log Monitoring via AugmentedSignal and a Hierarchical Recognizer. Ph.D. Thesis, Air University, Islamabad, Pakistan, December 2020.

Tahir SB, Jalal A, Kim K. IMU Sensor based Automatic-Features Descriptor for Healthcare Patient's daily life-log Recognition.

Uddin MZ, Thang ND, Kim JT, Kim T-S. 2011. Human activity recognition using body jointangle features and hidden Markov model. Etri Journal 33:569-579.

Ullah A, Muhammad K, Haq IU, Baik SW. 2019. Action recognition using optimized deep autoencoder and CNN for surveillance data streams of non-stationary environments. Future Generation Computer Systems. DOI: 10.1016/j.future.2019.01.029.

Umer R, Doering A, Leibe B, Gall J. 2020. Self-supervised keypoint correspondences for multi-person pose estimation and tracking in videos. arXiv preprint arXiv:2004.12652.

Viswakumar A, Rajagopalan V, Ray T, Parimi C. 2019. Human gait analysis using OpenPose. In: 2019 Fifth International Conference on Image Information Processing (ICIIP). 310-314.

Rehman MA, Raza H, Akhter I. 2018. SECURITY ENHANCEMENT OF HILL CIPHER BY USING NON-SQUARE MATRIX APPROACH. In: Proceedings of the 4th international

Peer) Comput. Sci. reviewing PDF | (CS-2021:04:60580:2:0:CHECK 8 Oct 2021) 
914

915

916

917

918

919

920

921

922

923

924

925

926

927

928

929

930

931

932

933

934

935

936

937

938

conference on knowledge and innovation in Engineering, Science and Technology. Acavent,. DOI: 10.33422/4kiconf.2018.12.24.

Wang Y, Du B, Shen Y, Wu K, Zhao G, Sun J, Wen H. 2019. EV-gait: Event-based robust gait recognition using dynamic vision sensors. In: Proceedings of the IEEE Computer Society Conference on Computer Vision and Pattern Recognition. DOI: 10.1109/CVPR.2019.00652.

Wang Y, Mori G. 2008. Multiple tree models for occlusion and spatial constraints in human pose estimation. In: Lecture Notes in Computer Science (including subseries Lecture Notes in Artificial Intelligence and Lecture Notes in Bioinformatics). DOI: 10.1007/978-3-54088690-7-53.

Wei S-E, Ramakrishna V, Kanade T, Sheikh Y. 2016. Multi-Person Articulated Tracking With Spatial and Temporal Embeddings,Proceedings of the IEEE/CVF Conference on Computer Vision . In: 2016 IEEE Conference on.

Yu J, Lei A, Hu Y. 2019. Soccer video event detection based on deep learning. In: Lecture Notes in Computer Science (including subseries Lecture Notes in Artificial Intelligence and Lecture Notes in Bioinformatics). DOI: 10.1007/978-3-030-05716-9_31.

Zheng Y, Gu S, Edelsbrunner H, Tomasi C, Benfey P. 2011. Detailed reconstruction of 3D plant root shape. In: 2011 International Conference on Computer Vision. 2026-2033.

Zhu Y, Zhou K, Wang M, Zhao Y, Zhao Z. 2019. A comprehensive solution for detecting events in complex surveillance videos. Multimedia Tools and Applications. DOI: 10.1007/s11042-018-6163-6.

Zou Y, Shi Y, Shi D, Wang Y, Liang Y, Tian Y. 2020. Adaptation-Oriented Feature Projection for One-shot Action Recognition. IEEE Transactions on Multimedia. DOI:

10.1109/tmm.2020.2972128. 
Figure 1

The proposed system model's structural design. 


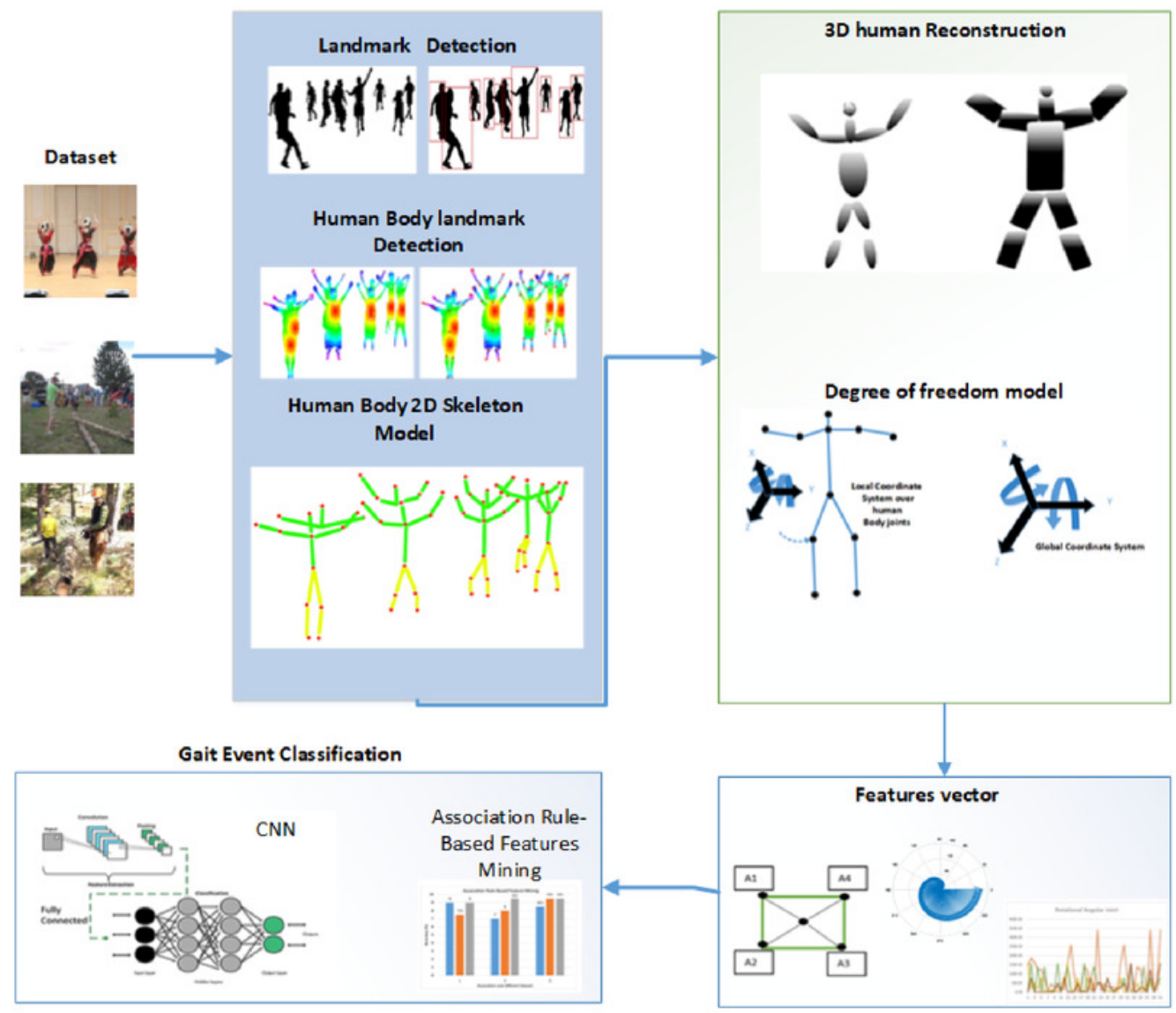


Figure 2

Flow chart of the proposed method. 


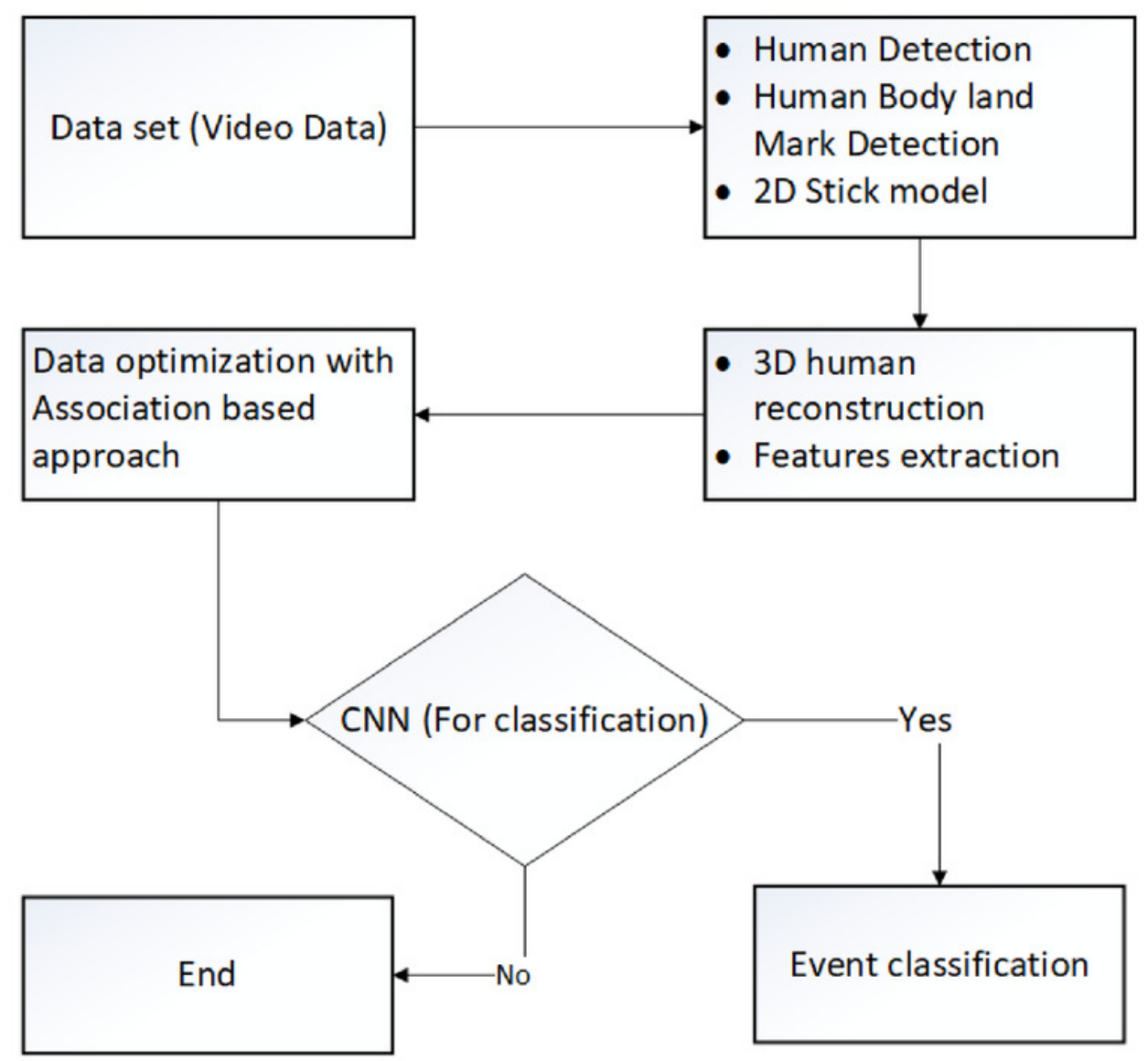




\section{Figure 3}

Results of different background subtraction techniques along with the original image. (a) Original image (b) change detection (c) floor detection (d) Markov random field and (e) spatial-temporal differencing. 


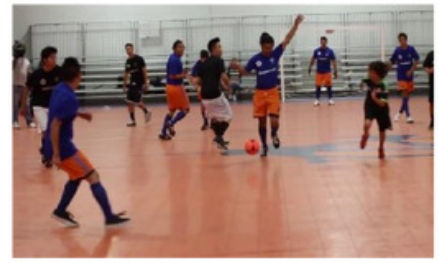

(a)

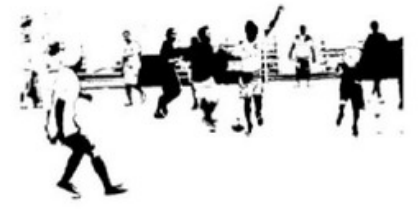

(b)

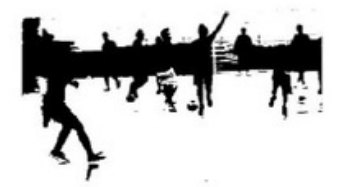

(d)

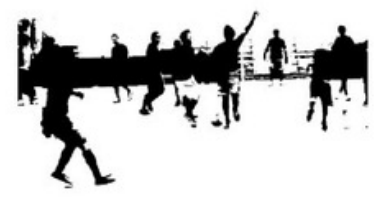

(c)

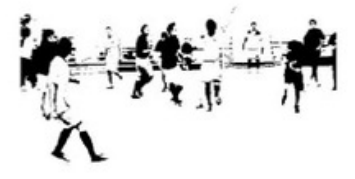

(e) 
Figure 4

Results of (a) Optimized human silhouette (b) human head detection (c) human detection in RGB videos and image sequences. 


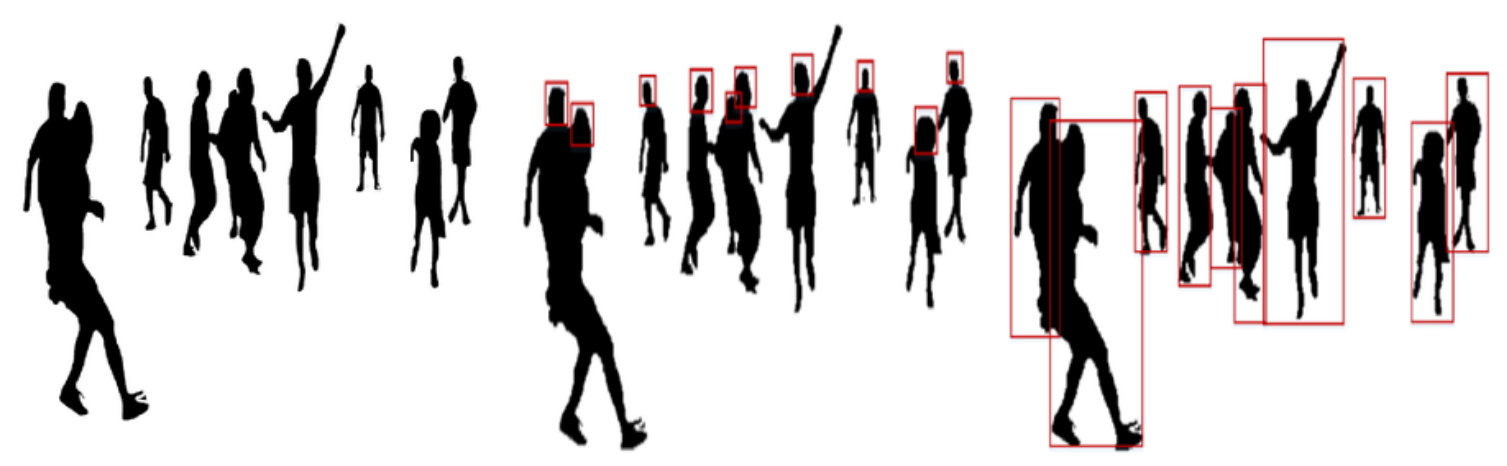




\section{Figure 5}

Human body landmark detection results (a) presents the landmark results using an HSV color map, (b) presents the eleven human body points. 

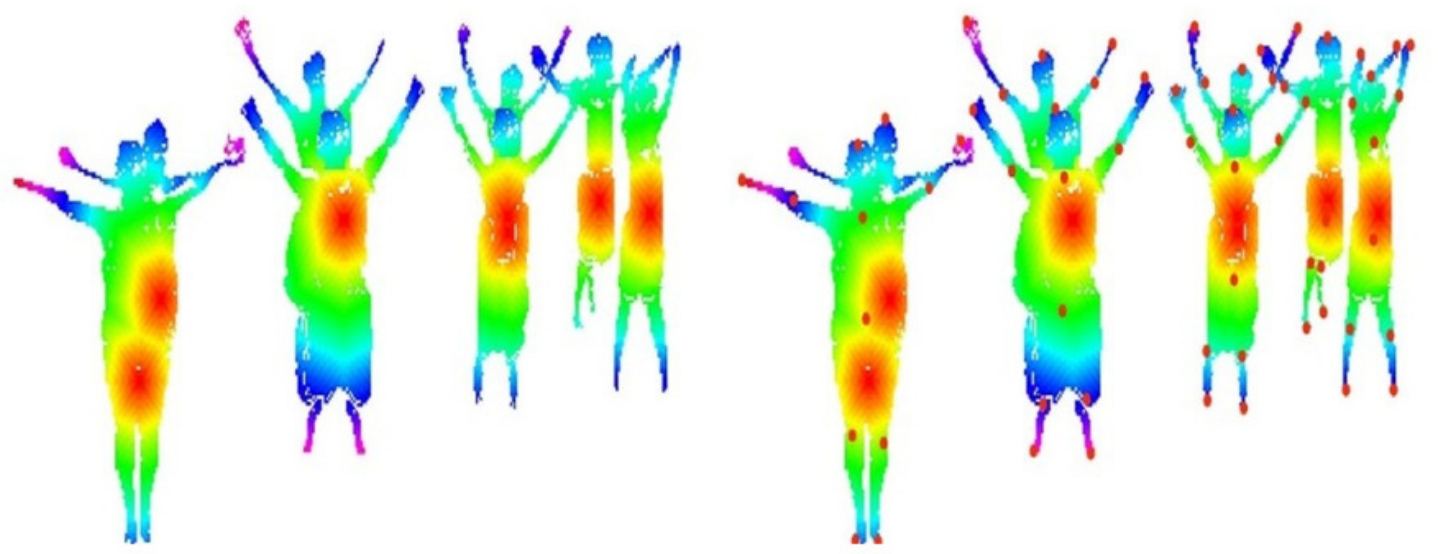


\section{Figure 6}

The Human 2D skeleton model results in over eleven human body parts. 


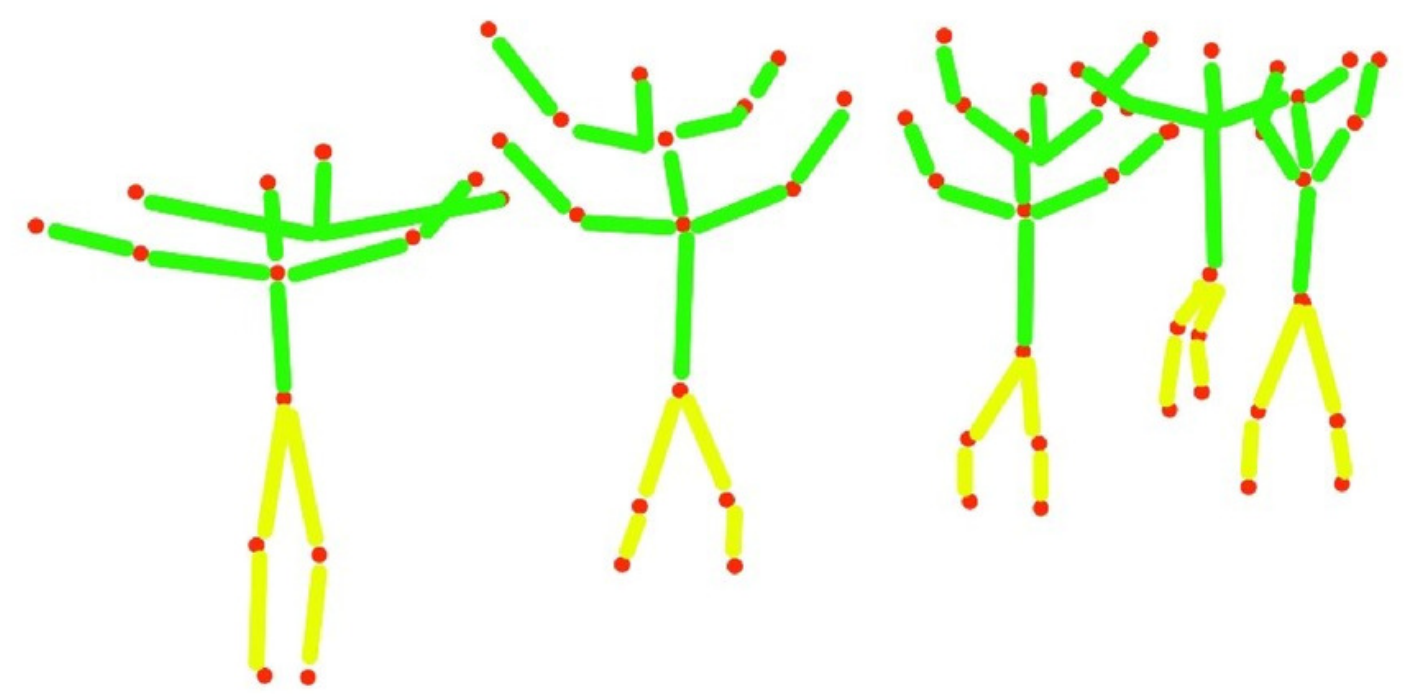




\section{Figure 7}

The results of the computational model with ellipsoids over human body points. 


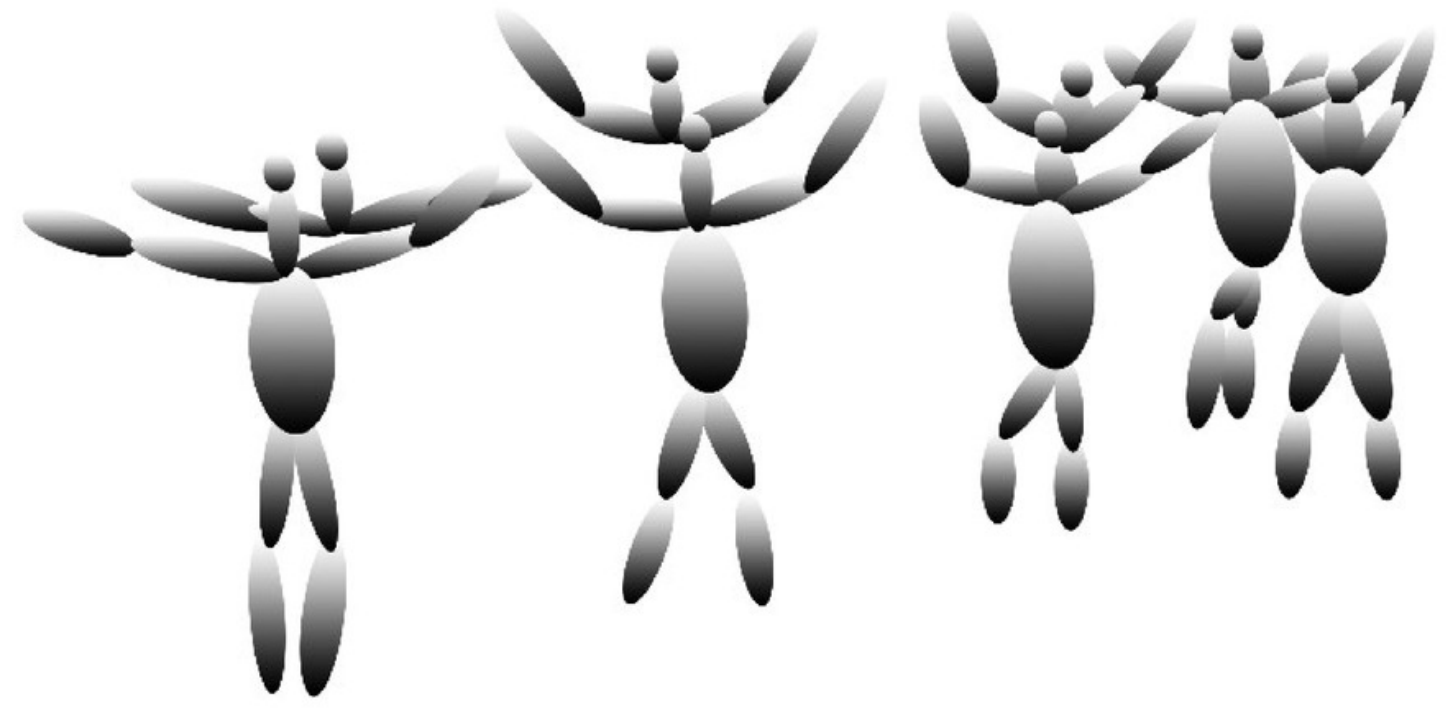




\section{Figure 8}

The results of the synthetic model with super quadrics over human body points. (a) Human 2D skeleton, (b) computational model with ellipsoids (c) synthetic model with super quadrics. 

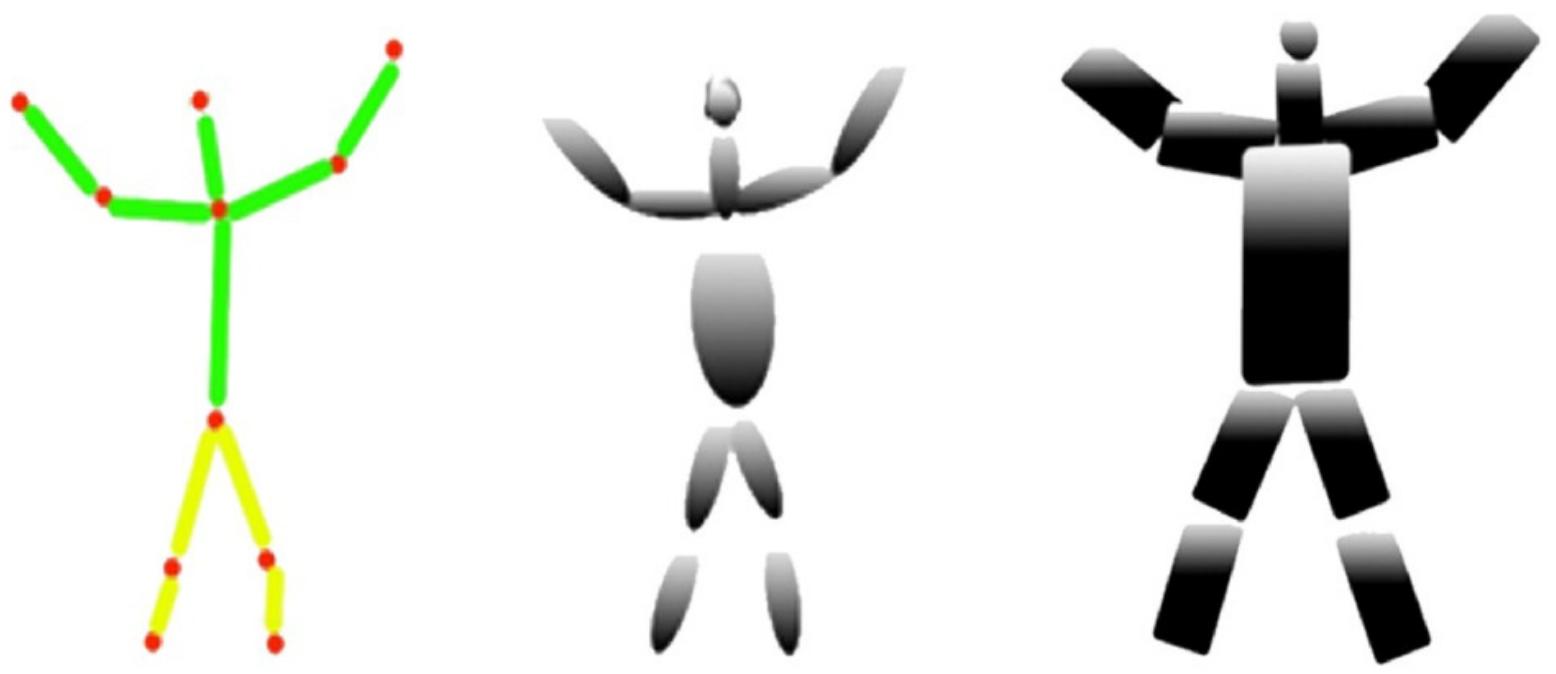
Figure 9

The theme concept of local and global coordinate systems. The left side shows the local coordinate system over the human left knee; the right side shows the DOF based global coordinate system. 

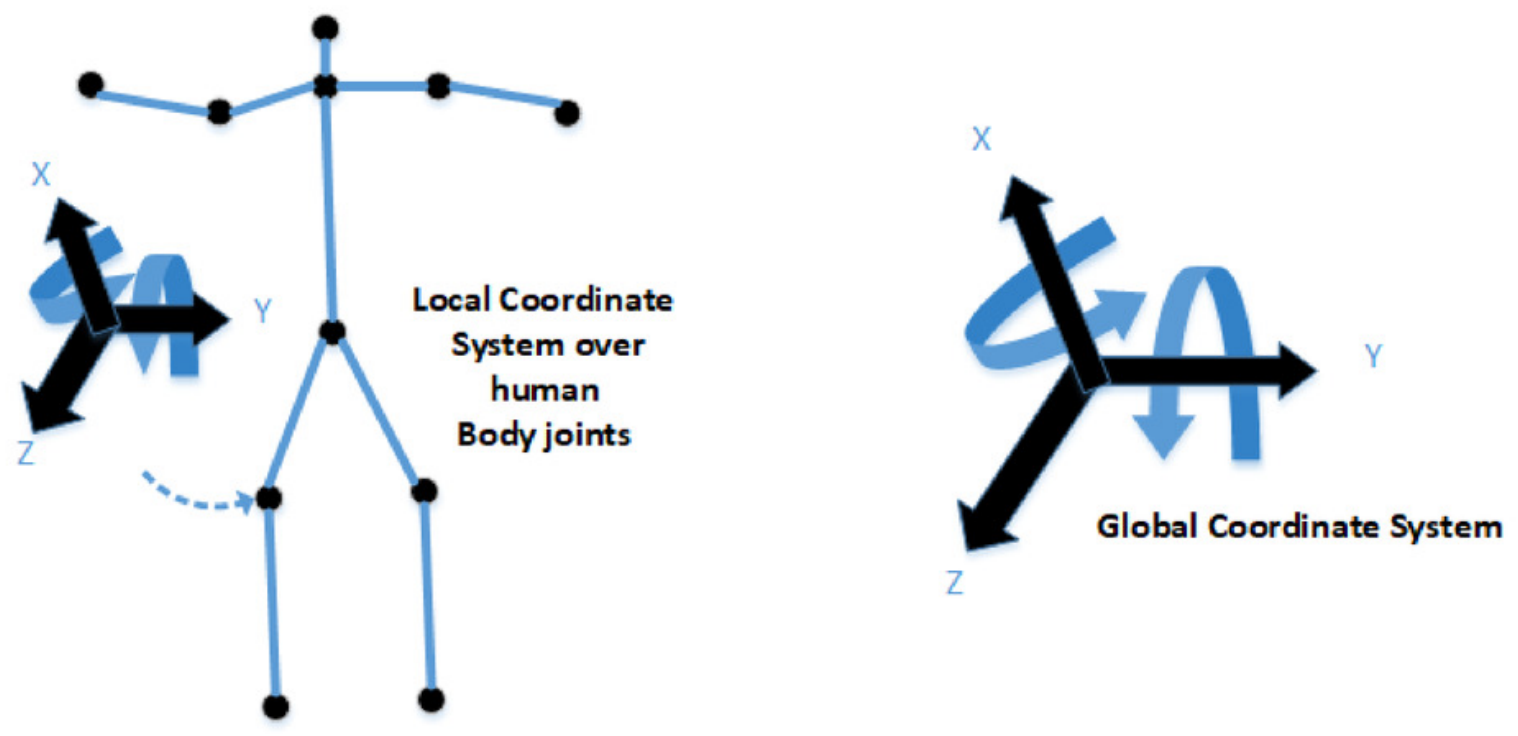


\section{Figure 10}

The results of the 3D ellipsoid reconstruction over the synthetic model with super quadrics and joint angle estimation 


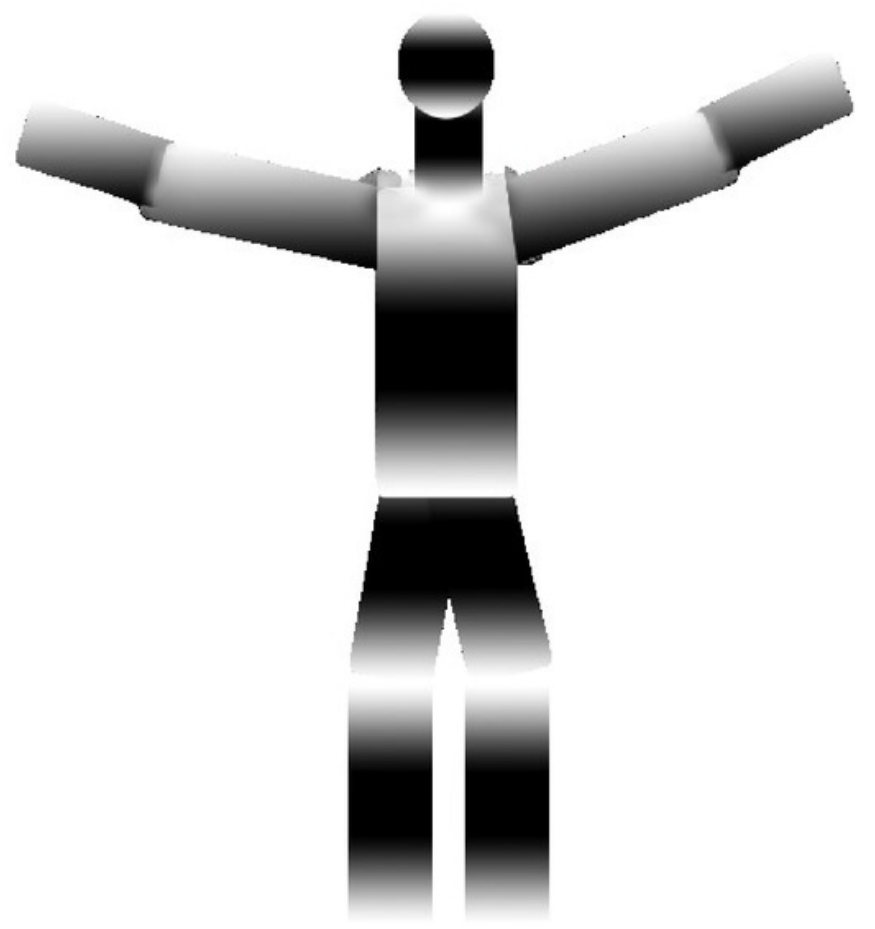


Figure 11

Few DOF results examples. 

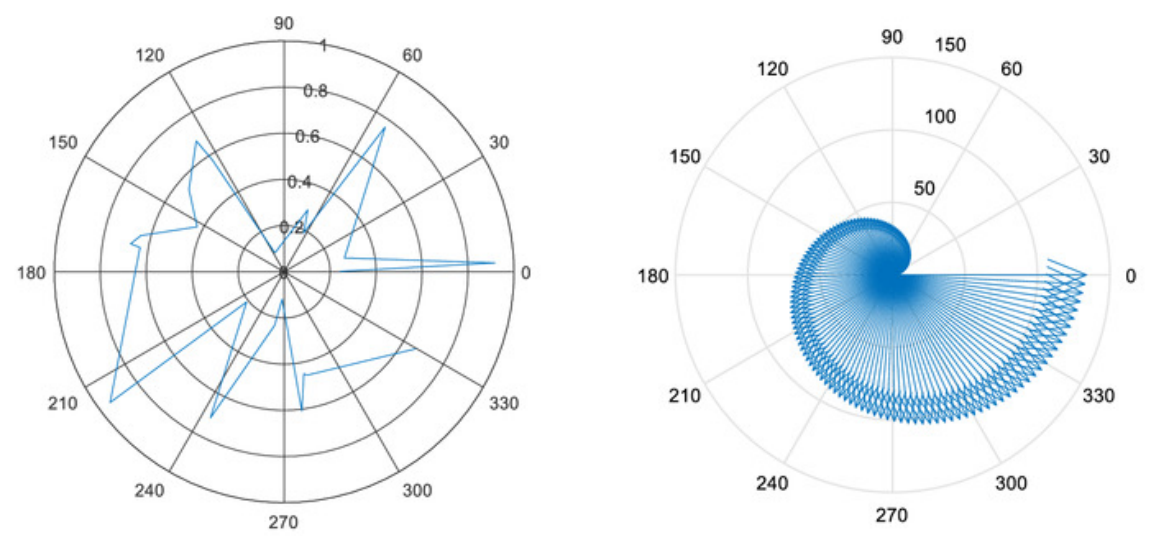
Figure 12

Periodic motion results. 


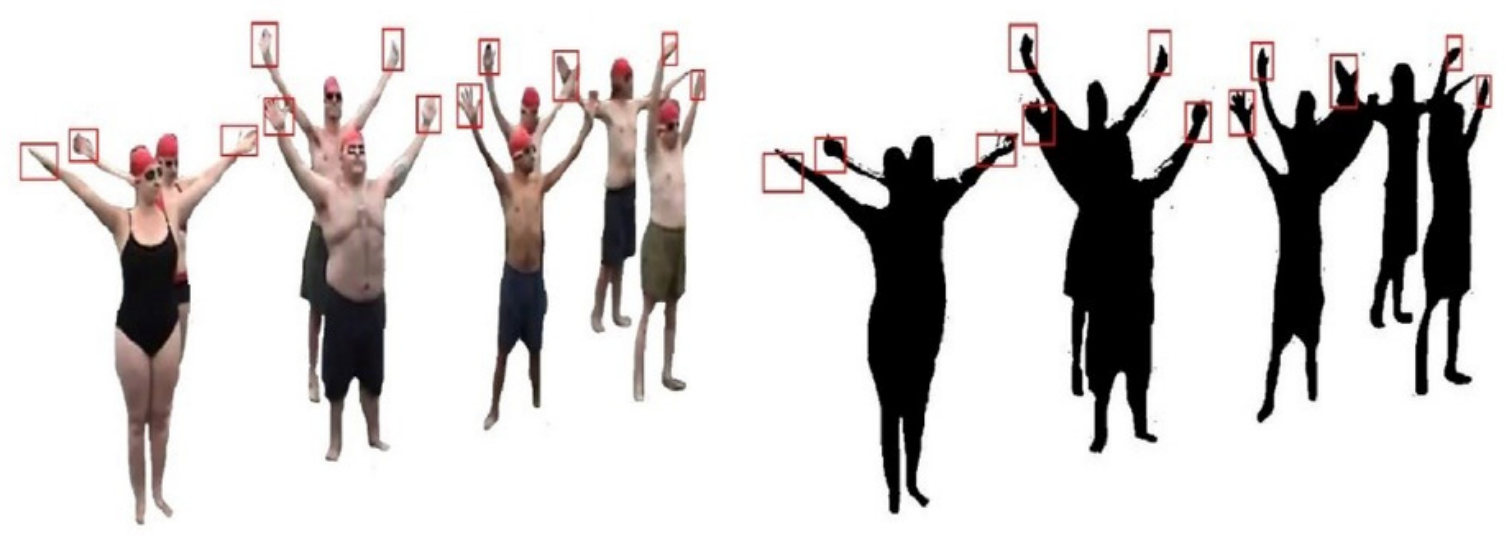


Figure 13

Results of non-periodic motion. 

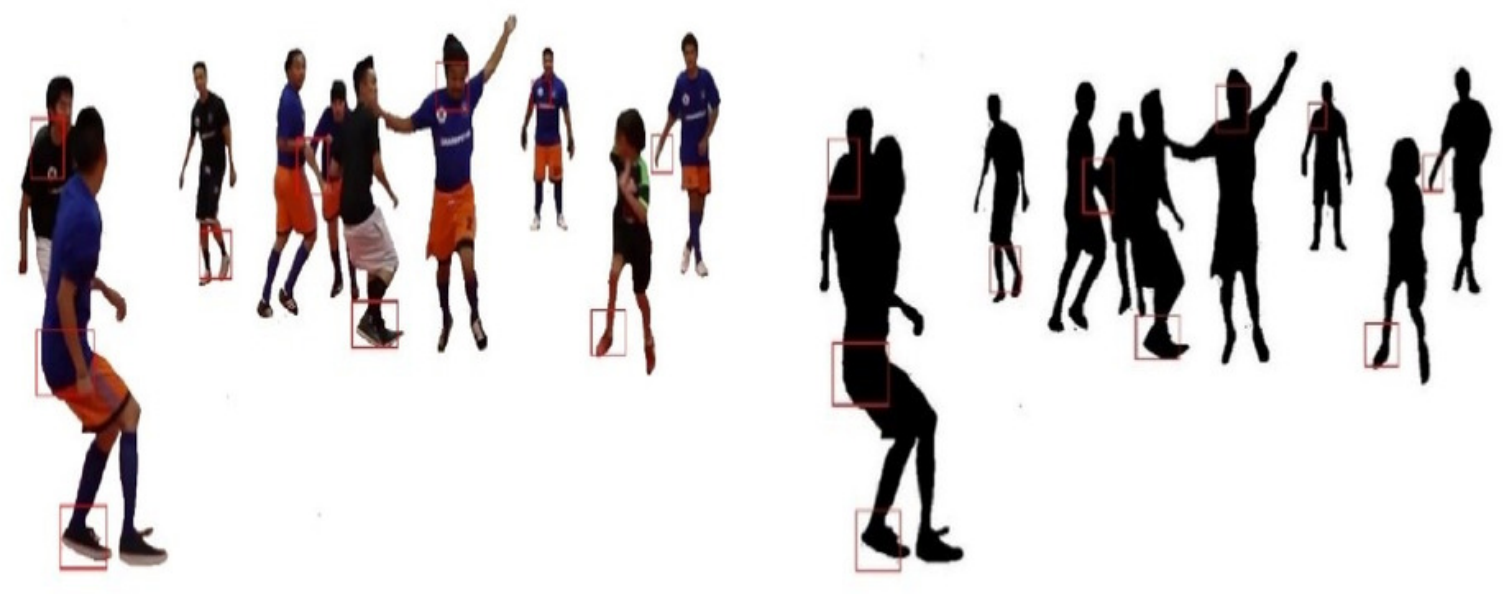
Figure 14

The results of motion direction flow over the basketball video. 

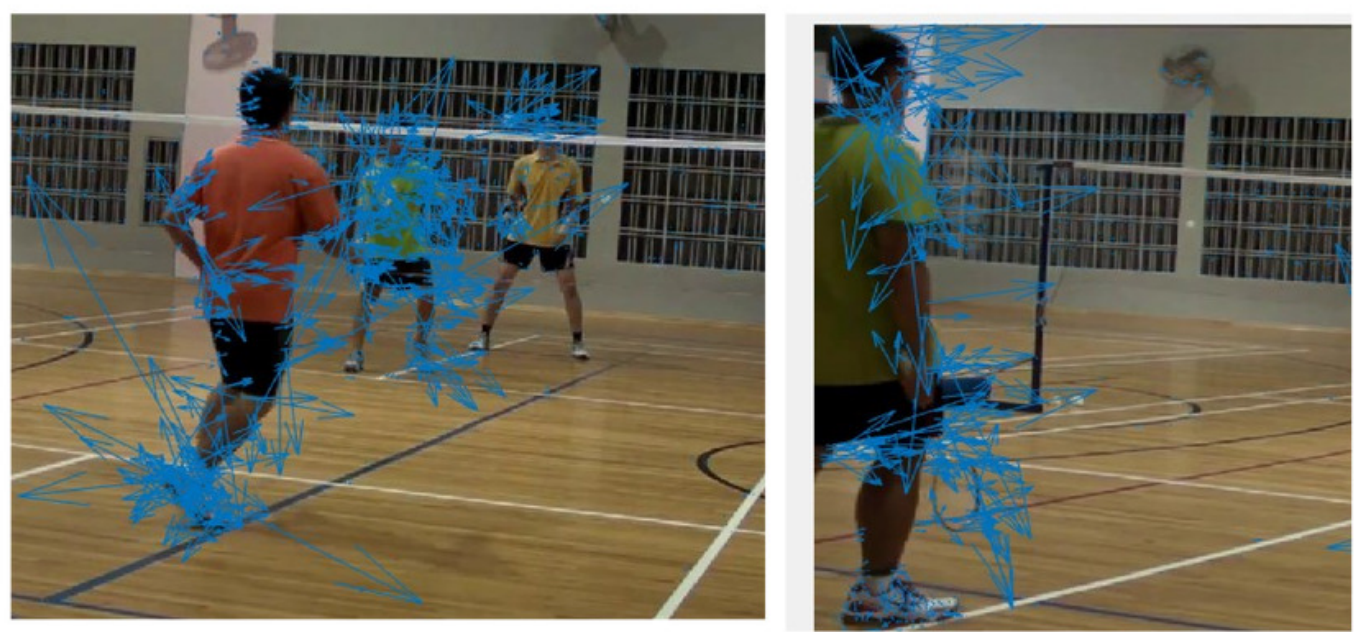


\section{Figure 15}

Rotational angular joint results and the pattern of rotational angels 

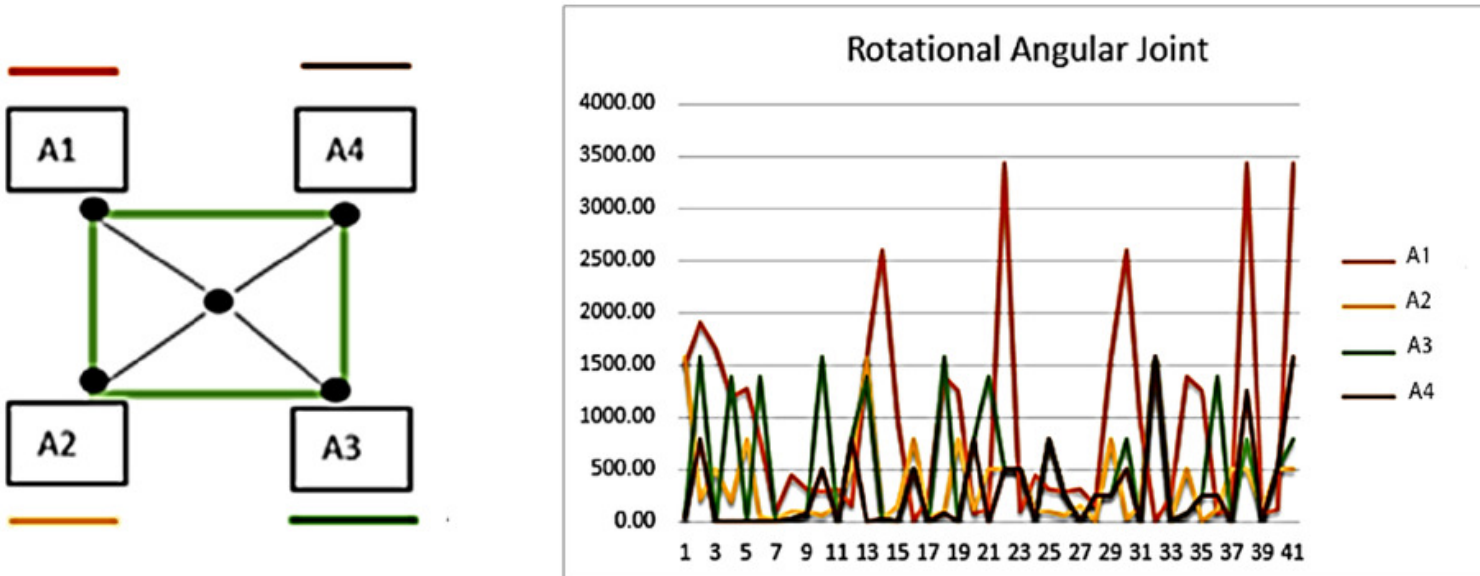


\section{Figure 16}

The most accurate features results via the rule-based features mining approach over the mpii-video pose, COCO, and Pose track datasets. 


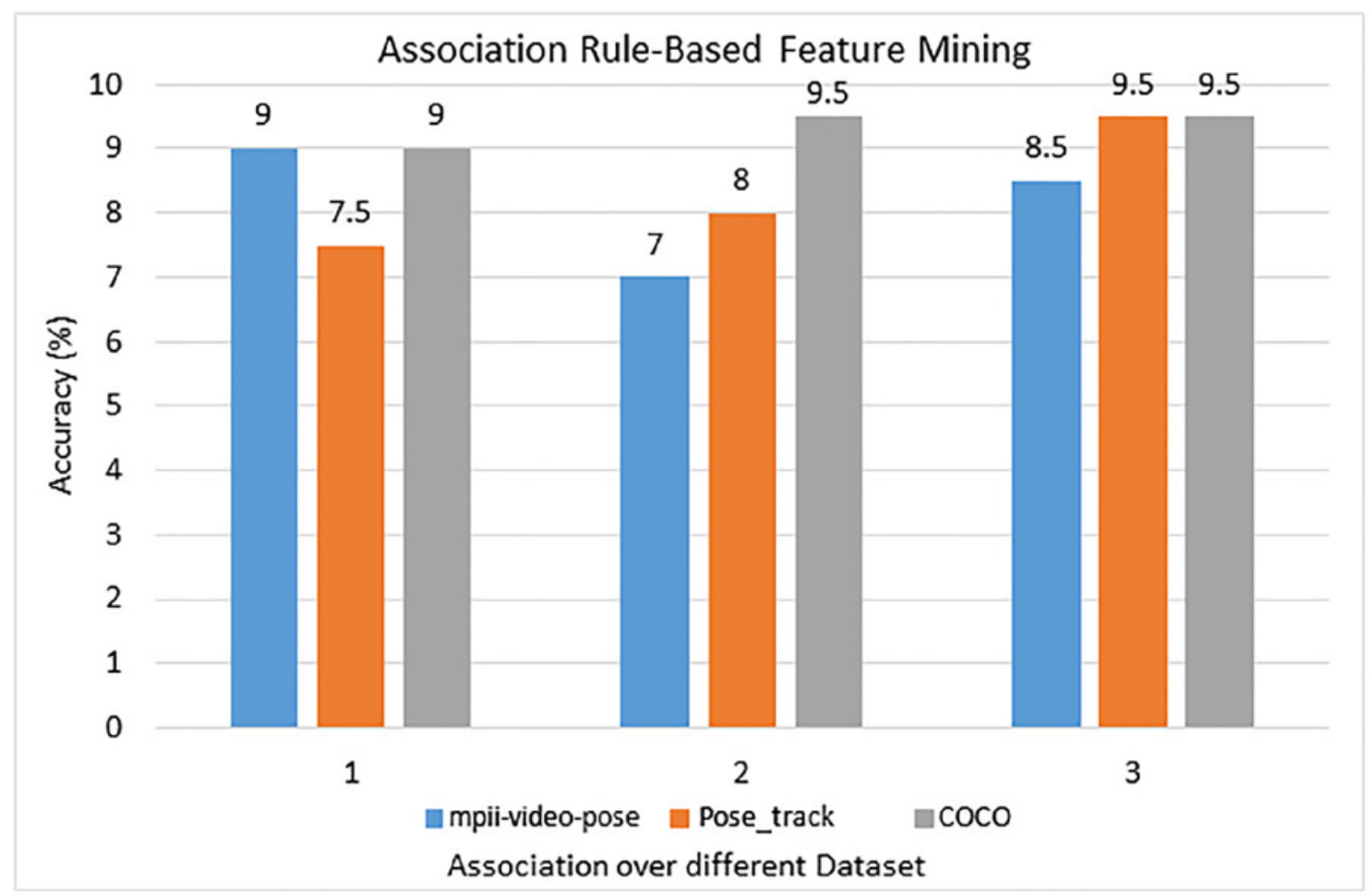


Figure 17

CNN model overview. 


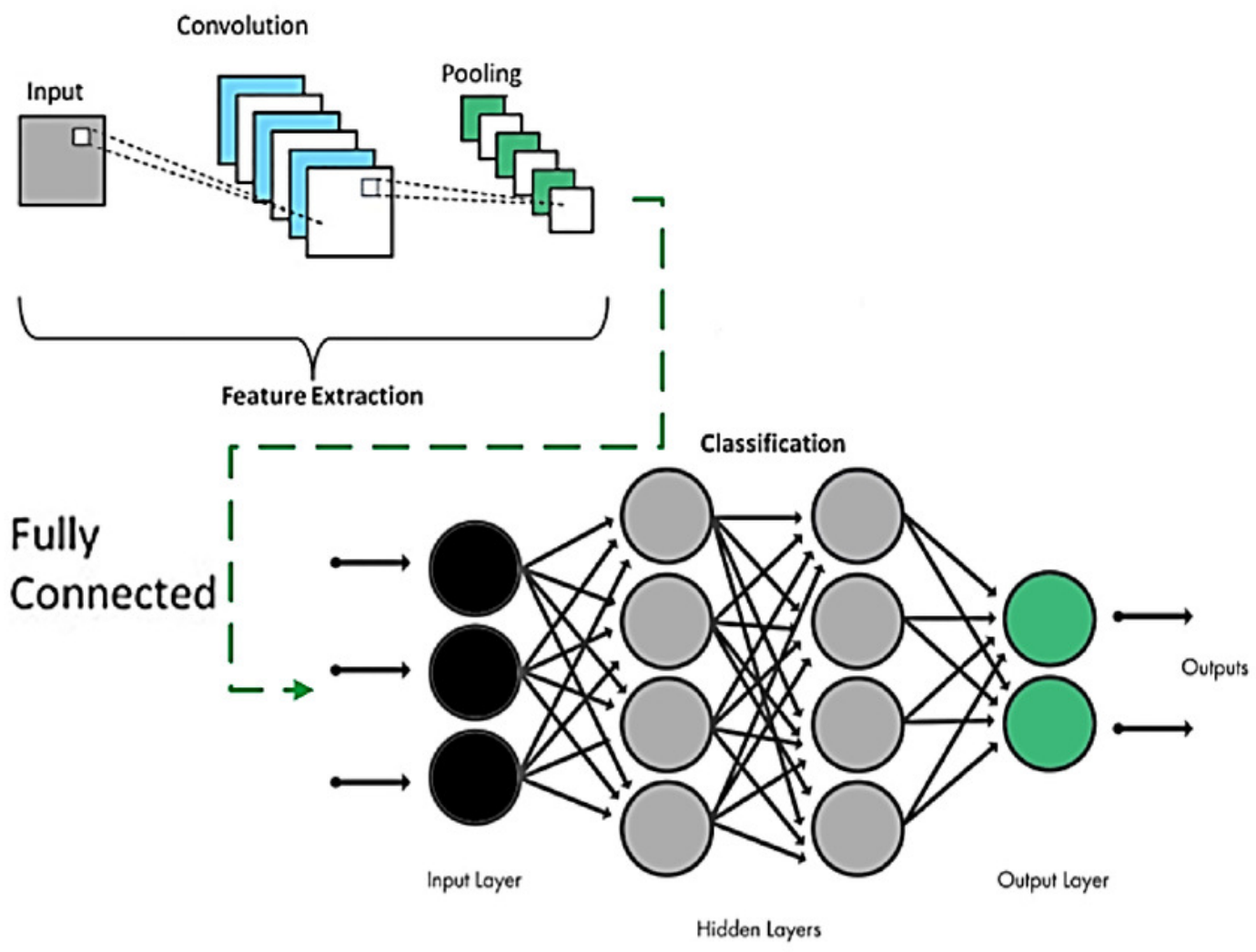




\section{Figure 18}

A few example images of the mpii-video-pose dataset. 

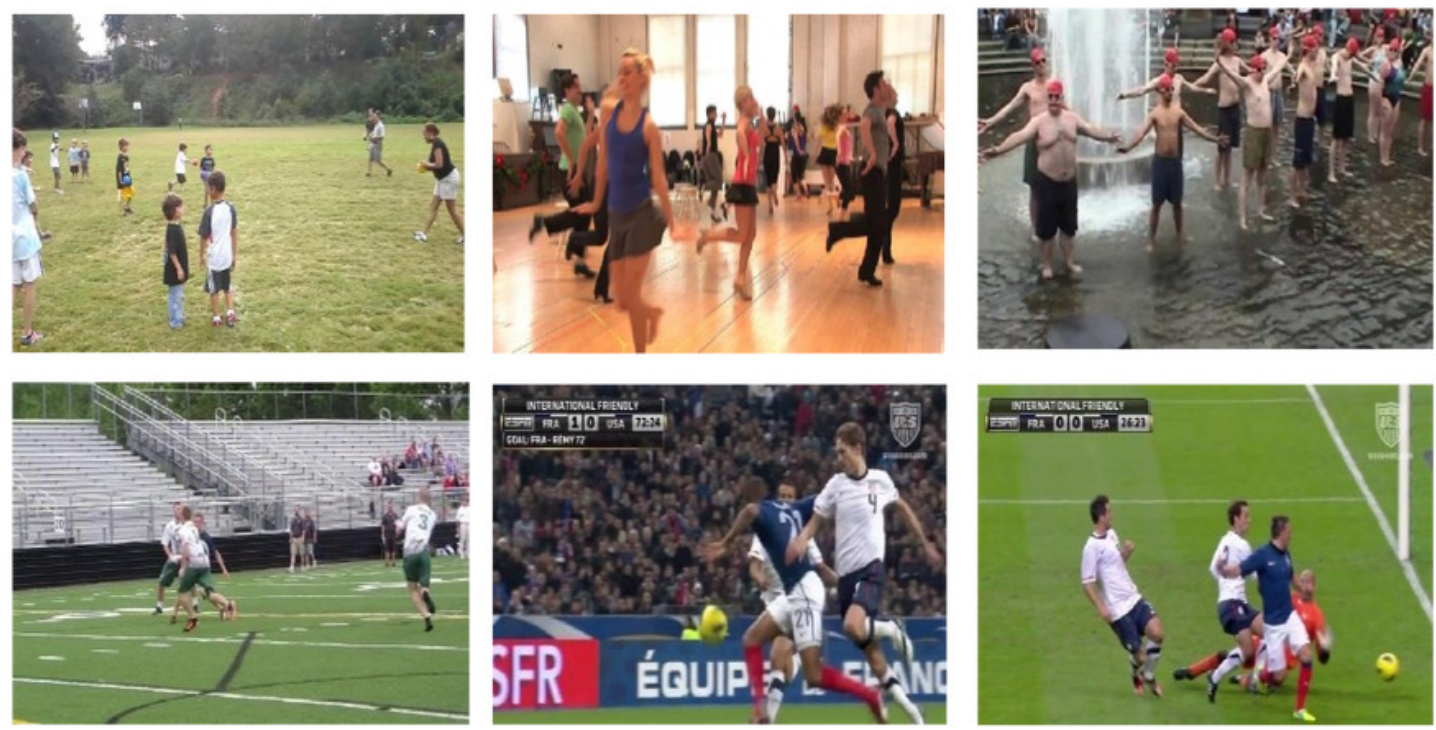
Figure 19

A few example images from the COCO dataset. 

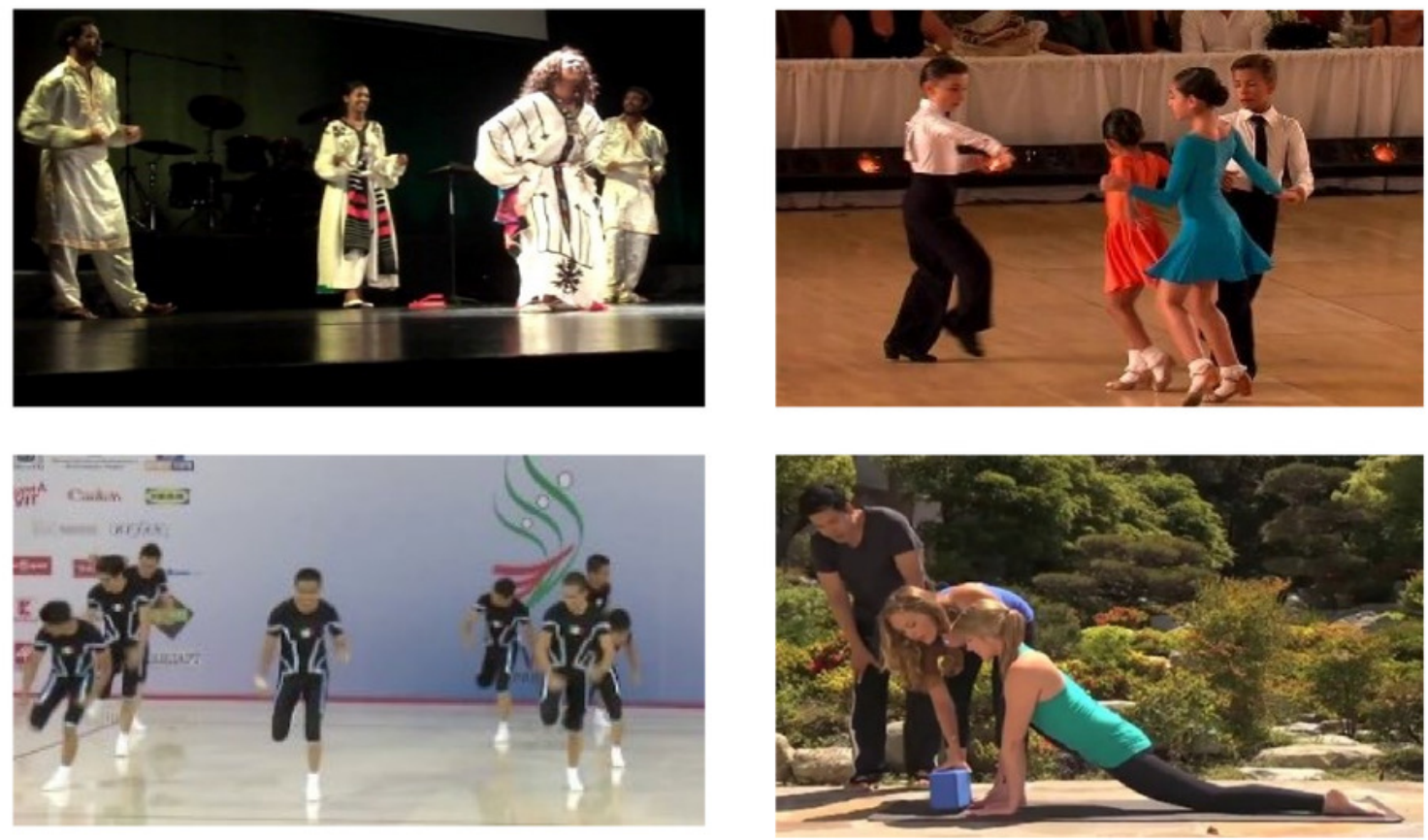
Figure 20

A few example images of the postmark dataset. 

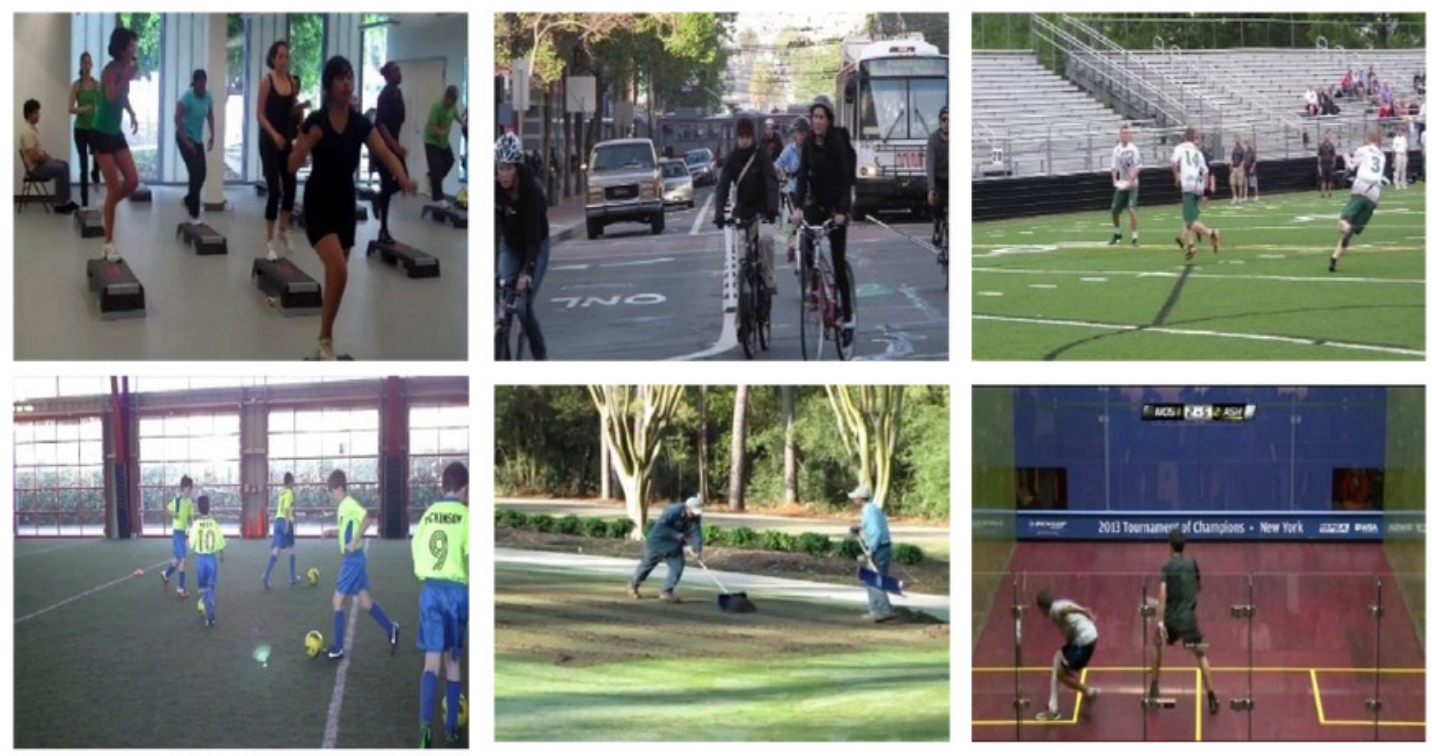
Figure 21

Confusion matrix results using CNN over Mpii-video-pose dataset. 


\begin{tabular}{llllllllllll}
\hline & $\mathrm{Bi}$ & $\mathrm{Ce}$ & $\mathrm{Da}$ & $\mathrm{Fh}$ & $\mathrm{Mp}$ & $\mathrm{Ra}$ & $\mathrm{Sp}$ & $\mathrm{Tr}$ & $\mathrm{Wl}$ & $\mathrm{Wa}$ & $\mathrm{Wn}$ \\
$\mathrm{Bi}$ & 9 & 1 & 0 & 0 & 0 & 0 & 0 & 0 & 0 & 0 & 0 \\
$\mathrm{Ce}$ & 0 & 9 & 0 & 0 & 0 & 0 & 1 & 0 & 0 & 0 & 0 \\
$\mathrm{Da}$ & 0 & 0 & 10 & 0 & 0 & 0 & 0 & 0 & 0 & 0 & 1 \\
$\mathrm{Fh}$ & 0 & 1 & 0 & 9 & 0 & 0 & 0 & 0 & 0 & 0 & 0 \\
$\mathrm{MP}$ & 1 & 0 & 0 & 0 & 9 & 0 & 0 & 0 & 0 & 0 & 0 \\
$\mathrm{Ra}$ & 0 & 0 & 1 & 0 & 0 & 9 & 0 & 0 & 0 & 0 & 0 \\
$\mathrm{SP}$ & 0 & 1 & 0 & 0 & 0 & 0 & 9 & 0 & 0 & 0 & 0 \\
$\mathrm{Tr}$ & 1 & 0 & 0 & 0 & 0 & 0 & 0 & 8 & 0 & 0 & 1 \\
$\mathrm{Wl}$ & 0 & 1 & 0 & 0 & 0 & 0 & 0 & 0 & 9 & 0 & 1 \\
$\mathrm{Wa}$ & 0 & 0 & 0 & 0 & 0 & 0 & 0 & 0 & 0 & 10 & 0 \\
$\mathrm{Wn}$ & 0 & 0 & 0 & 0 & 1 & 0 & 0 & 0 & 0 & 0 & 9 \\
& \multicolumn{7}{c}{ Gait event detection and classification mean accuracy $=\mathbf{9 0 . 9 0 \%}$} \\
\hline
\end{tabular}


Figure 22

Confusion matrix results using CNN over the COCO dataset. 


\begin{tabular}{llllllllllll}
\hline & $\mathrm{Bi}$ & $\mathrm{Da}$ & $\mathrm{Ce}$ & $\mathrm{Fh}$ & $\mathrm{Ra}$ & $\mathrm{Mp}$ & $\mathrm{Sp}$ & $\mathrm{Wl}$ & $\mathrm{Tr}$ & $\mathrm{Wa}$ & $\mathrm{Wn}$ \\
$\mathrm{Bi}$ & 8 & 0 & 0 & 0 & 1 & 0 & 0 & 0 & 1 & 0 & 0 \\
$\mathrm{Da}$ & 0 & 10 & 0 & 0 & 0 & 0 & 0 & 0 & 0 & 0 & 0 \\
$\mathrm{Ce}$ & 0 & 0 & 9 & 0 & 1 & 0 & 0 & 0 & 0 & 0 & 0 \\
$\mathrm{Fh}$ & 0 & 0 & 0 & 8 & 1 & 1 & 0 & 0 & 0 & 0 & 0 \\
$\mathrm{Ra}$ & 0 & 0 & 0 & 0 & 10 & 0 & 0 & 0 & 0 & 0 & 0 \\
$\mathrm{Mp}$ & 0 & 0 & 1 & 0 & 0 & 8 & 0 & 0 & 1 & 0 & 0 \\
$\mathrm{Sp}$ & 1 & 0 & 0 & 0 & 0 & 0 & 9 & 0 & 0 & 0 & 0 \\
$\mathrm{Wl}$ & 0 & 0 & 0 & 0 & 1 & 0 & 0 & 9 & 0 & 0 & 0 \\
$\mathrm{Tr}$ & 0 & 1 & 0 & 0 & 0 & 0 & 0 & 0 & 8 & 0 & 1 \\
$\mathrm{Wa}$ & 0 & 0 & 1 & 0 & 0 & 0 & 0 & 0 & 0 & 9 & 0 \\
$\mathrm{Wn}$ & 0 & 0 & 0 & 0 & 0 & 0 & 0 & 0 & 0 & 0 & 10 \\
& \multicolumn{7}{c}{ Gait event detection and classification mean accuracy $=\mathbf{8 9 . 0 9 \%}$} \\
\hline
\end{tabular}


Figure 23

Confusion matrix results using CNN over the Pose track dataset 


\begin{tabular}{llllllllllll}
\hline & $\mathrm{Ce}$ & $\mathrm{Bi}$ & $\mathrm{Da}$ & $\mathrm{Mp}$ & $\mathrm{Fh}$ & $\mathrm{Ra}$ & $\mathrm{Tr}$ & $\mathrm{Sp}$ & $\mathrm{Wl}$ & $\mathrm{Wn}$ & $\mathrm{Wa}$ \\
$\mathrm{Ce}$ & 7 & 0 & 0 & 1 & 0 & 0 & 1 & 0 & 1 & 0 & 0 \\
$\mathrm{Bi}$ & 0 & 8 & 1 & 0 & 0 & 0 & 0 & 0 & 0 & 1 & 0 \\
$\mathrm{Da}$ & 0 & 0 & 9 & 0 & 0 & 0 & 0 & 0 & 1 & 0 & 0 \\
$\mathrm{Mp}$ & 0 & 0 & 0 & 10 & 0 & 0 & 0 & 0 & 0 & 0 & 0 \\
$\mathrm{Fh}$ & 0 & 0 & 0 & 0 & 10 & 0 & 0 & 0 & 0 & 0 & 0 \\
$\mathrm{Ra}$ & 0 & 0 & 0 & 0 & 0 & 10 & 0 & 0 & 0 & 0 & 0 \\
$\mathrm{Tr}$ & 0 & 0 & 0 & 0 & 1 & 0 & 8 & 0 & 0 & 1 & 0 \\
$\mathrm{Sp}$ & 0 & 0 & 0 & 1 & 0 & 0 & 0 & 9 & 0 & 0 & 0 \\
$\mathrm{Wl}$ & 0 & 0 & 0 & 0 & 0 & 0 & 0 & 0 & 10 & 0 & 0 \\
$\mathrm{Wn}$ & 0 & 0 & 0 & 0 & 1 & 1 & 0 & 0 & 0 & 8 & 0 \\
$\mathrm{Wa}$ & 0 & 0 & 0 & 0 & 1 & 0 & 0 & 0 & 0 & 1 & 8 \\
& \multicolumn{7}{c}{ Gait event detection and classification mean accuracy $=\mathbf{8 8 . 1 8 \%}$} \\
\hline
\end{tabular}


Figure 24

Some examples of limitations and failure cases
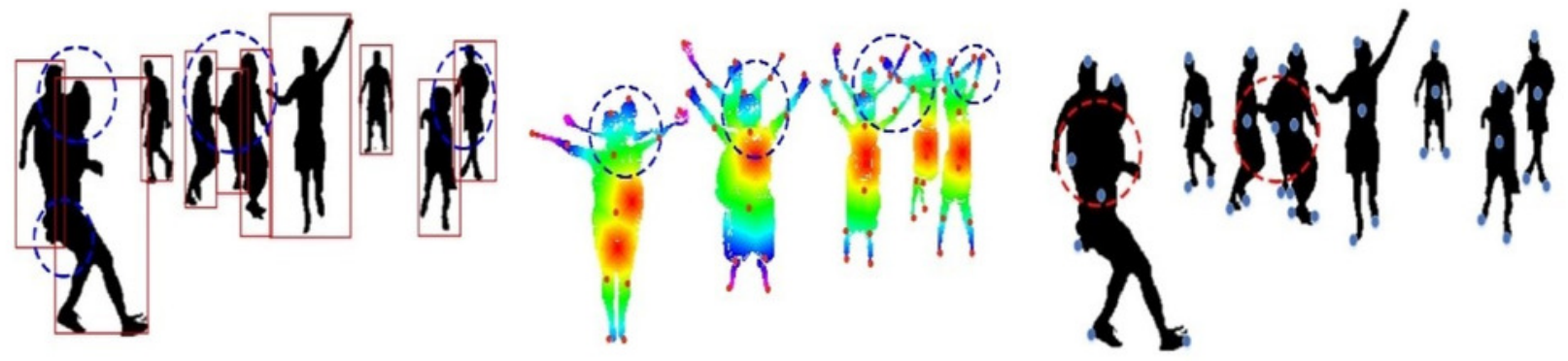
Figure 25

Results of the openpose CNN based classification 


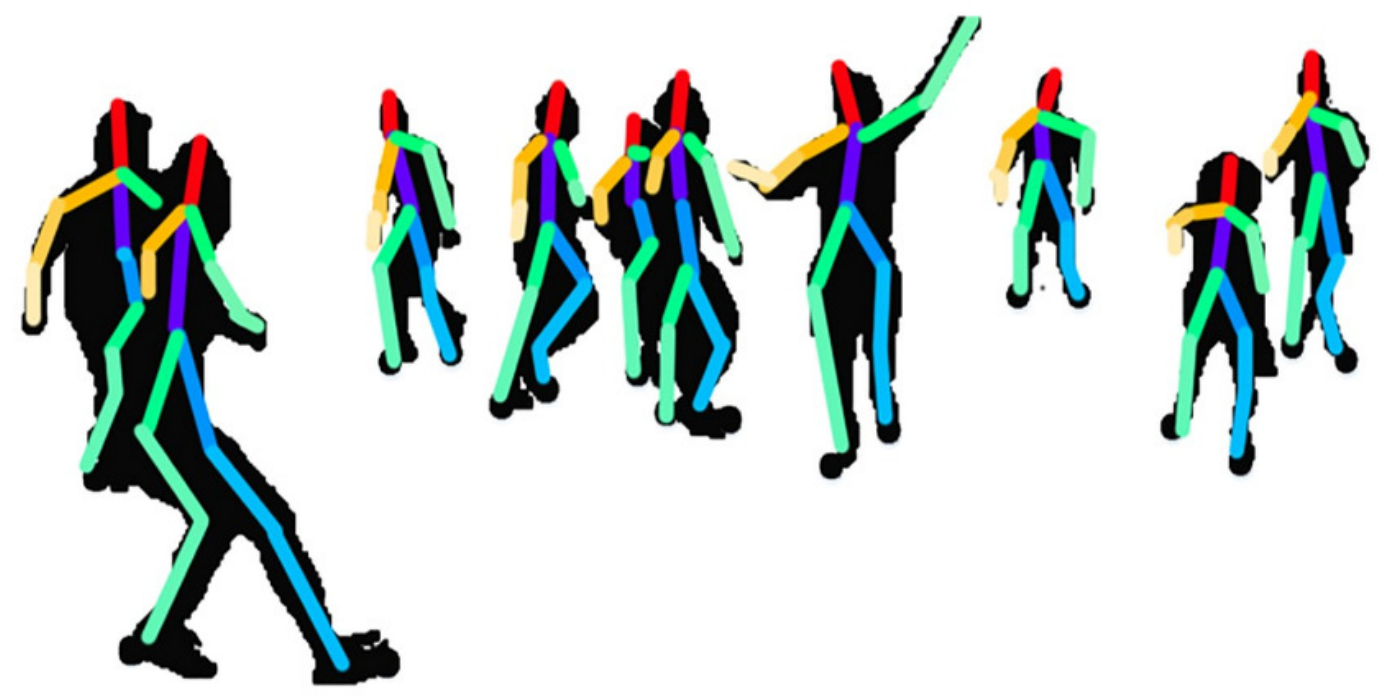




\section{Table 1 (on next page)}

Algo01 
Algorithm 1: Overview of the proposed method

Step 01. Input (Video based data set) Iv

Fun(Input)

Video_data1, Video_data2, Video_data3

Step 02. Preprocessing on video data Pd

Fun(Nosie_redu)

Fun(Frame_conv)

Fun(Gry_scale, Bin_Covn)

a. Noise Reeducation (Nr)

b. Frame conversion ( $\mathrm{Fc}$ )

c. Grey scale and binary conversion(Scb)

Step 03. Human body land mark detection()

Fun(Hum_Det)

Fun(Landmark_det)

$T_{H e}^{q} \leftarrow T_{H e}^{q-1}+\Delta T_{H e}^{q-1}$

Fun(Human_2D_Stick_model)

$U b p h=h \bowtie \ll n d S_{-} e \bowtie S_{-} h n$

$\mathrm{Mph}=\mathrm{S} \_t \perp U$ Ubph

$H L b s=h k \bowtie f \bowtie M p h$

Fun(3D_Human_recons)

$C_{m e}=\overline{P_{a}}\left(e_{x}, e_{y}\right) \overline{\mathbf{\square}} P_{a+1}\left(e_{x}, e_{y}\right.$

a. Human Detection (Hd)

b. Landmark detection(Ld)

c. Human 2D stick model(Hsm)

d. 3D Human reconstruction(Hr)

Step 04. Features extraction()

Fun(Peri_Motion)

$P M(t)=\alpha \sin (\omega t+k)$

Fun(Non_Peri_Motion)

$\operatorname{NPm}(t) \stackrel{=}{=} P_{t, t+1}^{-}-P_{t, t+2} \|$

Fun(Motion_Direction_Flow)

$M_{d f}=\sum_{0}^{p} I_{v l}(I) \rightarrow D$

Fun(Rotational_Angular_Joint)

$$
\begin{aligned}
& A 1=\cos (x, y) \rightarrow L, A 2=\cos (x, y) \rightarrow L \\
& A 3=\cos (x, y) \rightarrow L, A 4=\cos (x, y) \rightarrow L
\end{aligned}
$$

a. PeriodicMotion(Pm)

b. NonPeriodicMotion(mn)

c. MotionDirectionandFlow(Mdf)

d. RotationalAngularJoint(Raj)

Step 05. Data optimization(Association based approach)

Step 06. Event classification() 


$$
T R_{p}=\sum_{q} w i_{p . q} \times a_{p}+b_{q}
$$

Step 07. Output(Event Classification) Oc 


\section{Table 2 (on next page) \\ algo02}




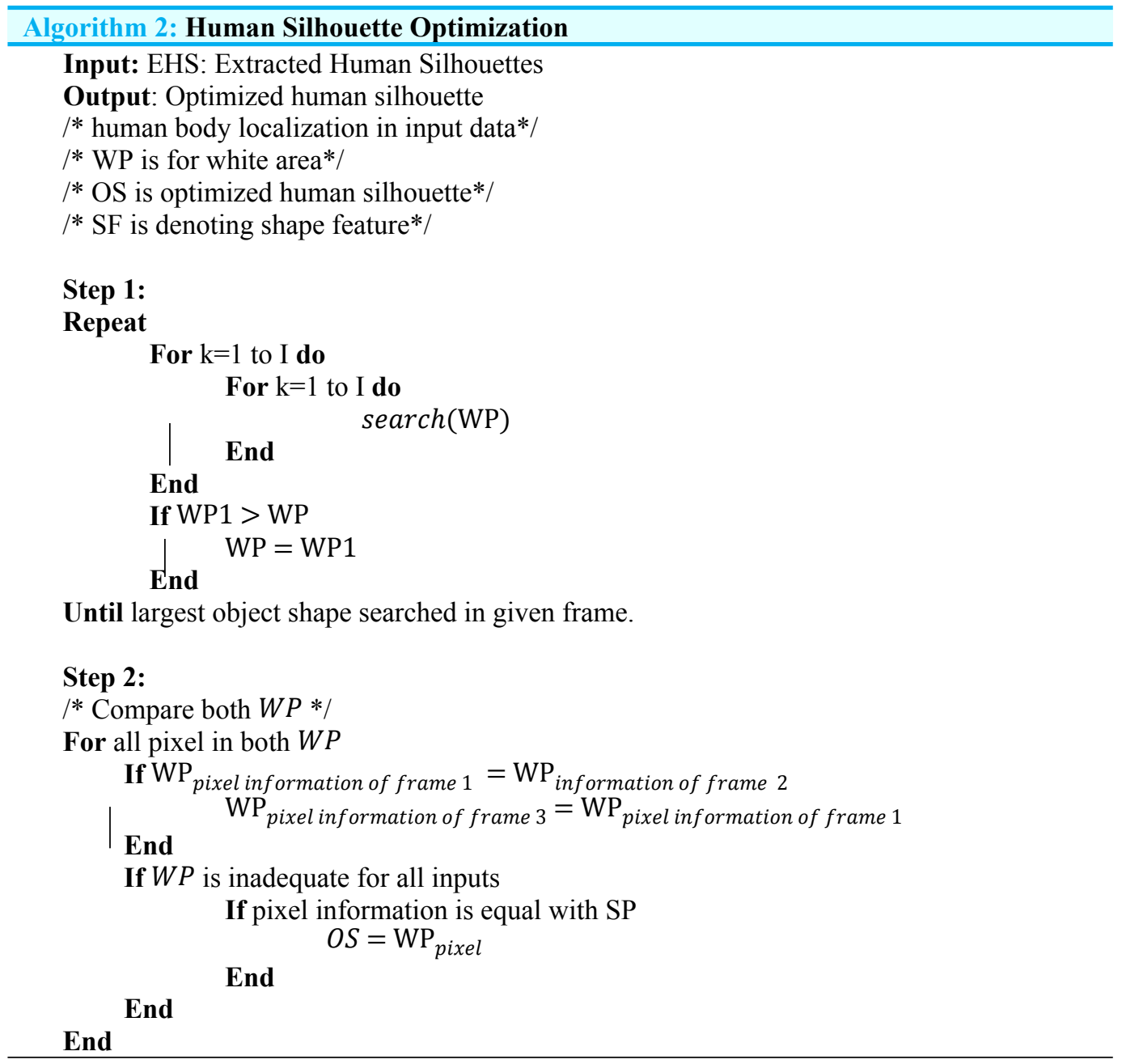




\section{Table 3 (on next page)}

Contextual Features Extraction 


\section{Algorithm 3: Contextual Features Extraction}

Input: HS: Human silhouette from RGB video data

Output: Contextual feature vectors $\left(C f_{1}, C f_{2}, C f_{3}, \ldots, C f_{n}\right)$

$\%$ feature vector for $\%$

Contextual_features_vec $\leftarrow$ []

CF_vecsize $\leftarrow$ GetFeaturesVectorsize ()

$\%$ loop over human silhouettes $\%$

For $\mathrm{i}=1: \mathrm{K}$

Contextual_features_vec_interactions $\leftarrow$ Get_Contextual_features_vec (interactions)

$\%$ extracting DOF, periodic motion, non periodic motion, motion direction and flow,

Rotational angular joint $\%$

$D O F \leftarrow$ ExtractDOF(Contextual_features_vec_interactions)

PeriodicMotion $\leftarrow$ ExtractPeriodicMotion (Contextual_features_vec _interactions)

NonPeriodicMotion $\leftarrow$ ExtractNonPeriodicMotion (Contextual_features_vec _interactions)

MotionDirectionandFlow $\leftarrow$ ExtractMotionDirectionandFlow(Contextual_features_vec_interactions)

RotationalAngularJoint $\leftarrow$ ExtractRotationalAngularJoint(Contextual_features_vec_interactions)

Contextualvectors $\leftarrow$ GetCFeaturevector

FVectors.append (CF_vectors)

End

Contextualvectors $\leftarrow$ Normalize (Contextual_features_vec)

return Contextual_features_vec $\left(C f_{1}, C f_{2}, C f_{3}, \ldots, C f_{n}\right)$ 


\section{Table 4 (on next page)}

Comprehensive review of relevant research. 
Table 1 Comprehensive review of relevant research. Human 2D posture analysis and event detection

Methods

Main contributions

\begin{tabular}{|c|c|}
\hline $\begin{array}{l}\text { Liu, Luo \& } \\
\text { Shah (2009) }\end{array}$ & $\begin{array}{l}\text { Using contextual, stationary, and vibration attributes, an effective randomized } \\
\text { forest-based methodology for human body part localization was developed. } \\
\text { They used videos and photographs to evaluate different human actions. }\end{array}$ \\
\hline $\begin{array}{c}\text { Khan et al. } \\
\text { (2020) }\end{array}$ & $\begin{array}{l}\text { A micro, horizontal, and vertical differential function was proposed as part of } \\
\text { an automated procedure. To classify human behavior, they used Deep Neural } \\
\text { Network (DNN) mutation. To accomplish DNN-based feature strategies, a pre- } \\
\text { trained Convolutional Neural Network Convolution layer was used. }\end{array}$ \\
\hline $\begin{array}{l}\text { Zou et al. } \\
\text { (2020) }\end{array}$ & $\begin{array}{l}\text { Adaptation-Oriented Features (AOF), an integrated framework with one-shot } \\
\text { image classification for approximation to human actions was defined. The } \\
\text { system applies to all classes, and they incorporated AOF parameters for } \\
\text { enhanced performance. }\end{array}$ \\
\hline $\begin{array}{l}\text { Franco, } \\
\text { Magnani \& } \\
\text { Maio (2020) }\end{array}$ & $\begin{array}{l}\text { They created a multilayer structure with significant human skeleton details using } \\
\text { RGB images. They used Histogram of Oriented (HOG) descriptor attributes to } \\
\text { identify human actions. }\end{array}$ \\
\hline $\begin{array}{l}\text { Ullah et al. } \\
\qquad \text { (2019) }\end{array}$ & $\begin{array}{l}\text { The defined a single Convolutional Neural Network (CNN)-based actual data } \\
\text { communications and information channel method. They utilized vision methods } \\
\text { to gather information through non-monitoring instruments. The Convolutional } \\
\text { Neural Network (CNN) technique is used to predict temporal features as well as } \\
\text { deep auto-encoders and deep features in order to monitor human behavior. }\end{array}$ \\
\hline $\begin{array}{l}\text { van der Kruk } \\
\qquad \& \text { Reijne } \\
\text { (2018) }\end{array}$ & $\begin{array}{l}\text { They developed an integrated approach to calculate vibrant human motion in } \\
\text { sports events using movement tracker sensors. The major contribution is the } \\
\text { computation of human events in sports datasets by estimating the kinematics of } \\
\text { human body joints, motion, velocity, and recreation of the human pose. }\end{array}$ \\
\hline $\begin{array}{c}\text { Wang \& Mori } \\
\text { (2008) }\end{array}$ & $\begin{array}{l}\text { They developed a lightweight event recognition strategy based on spatial } \\
\text { development and social body pose. The kinematics knowledge of attached } \\
\text { human body parts is used to characterize tree-based characteristics. }\end{array}$ \\
\hline $\begin{array}{l}\text { Amft \& } \\
\text { Tröster } \\
\text { (2008) }\end{array}$ & $\begin{array}{l}\text { Using a Hidden Markov methodology, they built a solid framework for event } \\
\text { identification which is accomplished using time-continuous dependent features } \\
\text { and body marker detectors. }\end{array}$ \\
\hline $\begin{array}{c}\text { Wang et al. } \\
\text { (2019) }\end{array}$ & $\begin{array}{l}\text { With the assistance of a human tracking methodology, they developed a } \\
\text { comprehensive new approach for estimating the accuracy of human motion. The } \\
\text { Deep Neural Network (DNN) is used to identify events. }\end{array}$ \\
\hline $\begin{array}{c}\text { Jiang et al. } \\
\text { (2015) }\end{array}$ & $\begin{array}{l}\text { They introduced a multidimensional function method for estimating human } \\
\text { motion and gestures. They used a late mean combination algorithm to recognize }\end{array}$ \\
\hline
\end{tabular}




\begin{tabular}{|c|c|}
\hline & lex scenes. \\
\hline $\begin{array}{l}\text { Li et al. } \\
\text { (2020) }\end{array}$ & $\begin{array}{l}\text { They developed a lightweight organizational approach focused on optimal } \\
\text { allocation, optical flow, and a histogram of the extracted optical flow. They were } \\
\text { able to achieve effective event recognition using the standard optimization } \\
\text { process, body joint restoration, and a Reduced and Compressed Coefficient } \\
\text { Dictionary Learning (LRCCDL) methodology. }\end{array}$ \\
\hline $\begin{array}{l}\text { Einfalt et al. } \\
\qquad \text { (2019) }\end{array}$ & $\begin{array}{l}\text { Through task identification, isolation of sequential } 2 \mathrm{D} \text { posture characteristics } \\
\text { and a convolutional sequence network, a coherent framework for event } \\
\text { recognition with athletes in motion was created. They correctly identified } \\
\text { number of sporting event. }\end{array}$ \\
\hline $\begin{array}{c}Y u, L e i \& H u \\
\text { (2019) }\end{array}$ & $\begin{array}{l}\text { Their work describes a probabilistic framework for detecting events in specific } \\
\text { interchanges in soccer rivalry videos. This is done using the replay recognition } \\
\text { approach which recognizes the most important background features for fulfilling } \\
\text { spectator needs and generating replay storytelling clips. }\end{array}$ \\
\hline $\begin{array}{l}\text { Franklin, } \\
\text { Mohana \& } \\
\text { Dabbagol } \\
\text { (2020) }\end{array}$ & $\begin{array}{l}\text { A comprehensive deep learning framework for identifying anomalous and } \\
\text { natural events was developed. The findings were obtained using differentiation, } \\
\text { grouping, and graph-based techniques. They discovered natural and unusual } \\
\text { features for event duration use using deep learning techniques. }\end{array}$ \\
\hline $\begin{array}{c}\text { Cao et. Al } \\
\text { (2019) }\end{array}$ & $\begin{array}{l}\text { This article is based on a real-time method for detecting the } 2 \mathrm{D} \text { posture of } \\
\text { numerous individuals in a picture. The suggested technique learns to connect } \\
\text { body parts with persons in the image using a nonparametric informed decision- } \\
\text { making Part Affinity Fields (PAFs). }\end{array}$ \\
\hline \multicolumn{2}{|c|}{ 3D human posture analysis and event detection } \\
\hline $\begin{array}{c}\text { Aggarwal \& } \\
\text { Cai (1999) }\end{array}$ & $\begin{array}{l}\text { They devised a reliable method for analyzing the movement of human body } \\
\text { parts through multiple cameras which monitor the body parts detection. They } \\
\text { also created a simulation for human body joints that is } 2 \mathrm{D}-3 \mathrm{D} \text {. }\end{array}$ \\
\hline $\begin{array}{c}\text { Hassner \& } \\
\text { Basri (2006) }\end{array}$ & $\begin{array}{l}\text { They designed an example-based synthesis methodology using a single class- } \\
\text { based objects database that holds example reinforcements of realistic mappings } \\
\text { due to the complexity of the objects. }\end{array}$ \\
\hline $\begin{array}{c}\text { Hu et al. } \\
\text { (2004) }\end{array}$ & $\begin{array}{l}\text { To define facial dimensionality, an effective 2D-to-3D hybrid face } \\
\text { reconstruction technique is used to recreate a customizable 3D face template } \\
\text { from a single cortical face picture with a neutral expression and regular lighting. } \\
\text { Immersive-looking faces including different PIE are synthesized based on the } \\
\text { customizable 3D image. }\end{array}$ \\
\hline $\begin{array}{l}\text { Zheng et al. } \\
\text { (2011) }\end{array}$ & $\begin{array}{l}\text { To enhance the classification of both the roots from each } 2 \mathrm{D} \text { image, they initially } \\
\text { model the context only as a harmonic function. Second, they analyze the }\end{array}$ \\
\hline
\end{tabular}




\begin{tabular}{|c|c|}
\hline & $\begin{array}{l}\text { formalized graphical hull definition, which eliminates jitter and diffusion by } \\
\text { maintaining continuity with a single } 2 \mathrm{D} \text { image. Third, they maintain } \\
\text { connectivity by making variations to the } 3 \mathrm{D} \text { reconstruction by global errors } \\
\text { minimization. }\end{array}$ \\
\hline $\begin{array}{l}\text { Uddin et al. } \\
\text { (2011) }\end{array}$ & $\begin{array}{l}\text { They proposed a heuristic approach for human activity detection and human } \\
\text { posture analysis. For this, they utilized human body joint angle information with } \\
\text { the help of the hidden Markov model (HMM). }\end{array}$ \\
\hline $\begin{array}{l}\text { Lohithashva, } \\
\text { Aradhya \& } \\
\text { Guru(2020) }\end{array}$ & $\begin{array}{l}\text { The researchers created a deep learning system for detecting abnormal and } \\
\text { normal events. Distinction, classification, and graph-based methods were used } \\
\text { to obtain the results. Using deep learning methods, they explored natural and } \\
\text { uncommon features for event interval use. }\end{array}$ \\
\hline $\begin{array}{c}\text { Feng et al. } \\
\text { (2020) }\end{array}$ & $\begin{array}{l}\text { To retrieve deep features' spatial locations in composite images, a guided Long } \\
\text { Short-Term Memory (LSTM) approach that is based on a Convolutional Neural } \\
\text { Network (CCN) system was evaluated. For personal authentication, the state-of- } \\
\text { the-art YOLO v3 template was used and, for event recognition, a directed Long } \\
\text { Short-Term Memory (LSTM) driven method was used. }\end{array}$ \\
\hline $\begin{array}{l}\text { Khan et al. } \\
\text { (2020) }\end{array}$ & $\begin{array}{l}\text { They developed home-based patient control strategies based on body-marker } \\
\text { detectors. To record data from patients, body-marker sensors with a color } \\
\text { indicator framework are connected to the joints. }\end{array}$ \\
\hline $\begin{array}{l}\text { Mokhlespour } \\
\text { Esfahani et } \\
\text { al. (2017) }\end{array}$ & $\begin{array}{l}\text { For sporting events, human movement monitoring body-marker tools were used } \\
\text { to establish a Trunk Motion Method (TMM) with Body-worn Sensors that } \\
\text { provide a low power physical system (BWS). Twelve removable detectors were } \\
\text { used to measure 3D trunk movements in this process. }\end{array}$ \\
\hline $\begin{array}{l}\text { Golestani \& } \\
\text { Moghaddam,( } \\
\text { 2020) }\end{array}$ & $\begin{array}{l}\text { A robust wireless strategy was developed for detecting physical human } \\
\text { behavior. They used a magnetic flux cable to monitor human behavior, and } \\
\text { thematic maps were attached to the body joints. Research lab approximation } \\
\text { function and Deep RNN (Recurrent Neural Network) were used to enhance } \\
\text { efficiency. }\end{array}$ \\
\hline
\end{tabular}




\section{Table 5 (on next page)}

Human body parts recognition and detection accuracy 
Table 2: Human body parts recognition and detection accuracy

\begin{tabular}{ccccccc}
$\begin{array}{c}\text { Body key } \\
\text { points }\end{array}$ & Distance & $\begin{array}{c}\text { MPII } \\
\mathbf{( \% )}\end{array}$ & Distance & COCO (\%) & Distance & $\begin{array}{c}\text { Posetrack } \\
\mathbf{( \% )}\end{array}$ \\
\hline HP & 11.2 & 88 & 9.70 & 88 & 9.90 & 91 \\
NP & 10.8 & 86 & 10.2 & 86 & 11.1 & 88 \\
REP & 11.5 & 82 & 10.1 & 83 & 14.1 & 86 \\
RHP & 12.1 & 81 & 11.7 & 82 & 12.7 & 83 \\
LEP & 11.1 & 83 & 11.9 & 79 & 11.0 & 88 \\
LHP & 12.0 & 77 & 11.7 & 81 & 12.0 & 79 \\
MP & 10.1 & 91 & 13.1 & 90 & 11.9 & 91 \\
LKP & 13.2 & 94 & 12.8 & 92 & 12.3 & 87 \\
RKP & 9.90 & 91 & 10.3 & 91 & 11.7 & 81 \\
LFP & 10.3 & 94 & 11.2 & 95 & 14.1 & 94 \\
RFP & 11.5 & 91 & 10.3 & 94 & 13.8 & 97 \\
Mean Accuracy Rate & $\mathbf{8 7 . 0 9}$ & & $\mathbf{8 7 . 3 6}$ & & $\mathbf{8 7 . 7 2}$ \\
\hline
\end{tabular}

1 


\section{Table 6 (on next page)}

Human body parts results of multi-person for mpii-video-pose dataset 


\begin{tabular}{|c|c|c|c|c|c|}
\hline \multicolumn{6}{|c|}{ Table 3: Human body parts results of multi-person for mpii-video-pose dataset } \\
\hline Body parts & Human1 & Human2 & Human3 & Human4 & Human5 \\
\hline HP & $\checkmark$ & $\checkmark$ & $\checkmark$ & $\checkmark$ & $x$ \\
\hline NP & $x$ & $\checkmark$ & $x$ & $x$ & $\checkmark$ \\
\hline REP & $\checkmark$ & $\checkmark$ & $\checkmark$ & $\checkmark$ & $\checkmark$ \\
\hline RHP & $\checkmark$ & $x$ & $x$ & $\checkmark$ & $\checkmark$ \\
\hline LEP & $x$ & $\checkmark$ & $\checkmark$ & $\checkmark$ & $x$ \\
\hline LHP & $x$ & $\checkmark$ & $\checkmark$ & $x$ & $\checkmark$ \\
\hline MP & $\checkmark$ & $x$ & $\checkmark$ & $\checkmark$ & $\checkmark$ \\
\hline LKP & $\checkmark$ & $\checkmark$ & $x$ & $x$ & $\checkmark$ \\
\hline RKP & $x$ & $x$ & $\checkmark$ & $\checkmark$ & $x$ \\
\hline LFP & $\checkmark$ & $\checkmark$ & $x$ & $\checkmark$ & $\checkmark$ \\
\hline RFP & $\checkmark$ & $\checkmark$ & $\checkmark$ & $\checkmark$ & $\checkmark$ \\
\hline Accuracy & $63.63 \%$ & $72.72 \%$ & $63.63 \%$ & $72.72 \%$ & $72.72 \%$ \\
\hline \multicolumn{6}{|c|}{ Mean accuracy $=69.09 \%$} \\
\hline
\end{tabular}

1 


\section{Table 7 (on next page)}

Human body parts results of multi-person for COCO dataset 


\begin{tabular}{|c|c|c|c|c|c|}
\hline \multicolumn{6}{|c|}{ Table 4: Human body parts results of multi-person for COCO dataset } \\
\hline Body parts & Human 1 & Human2 & Human3 & Human4 & Human5 \\
\hline HP & $\checkmark$ & $\checkmark$ & $\checkmark$ & $\checkmark$ & $\checkmark$ \\
\hline NP & $\checkmark$ & $\checkmark$ & $\checkmark$ & $x$ & $\checkmark$ \\
\hline REP & $\checkmark$ & $x$ & $x$ & $\checkmark$ & $\checkmark$ \\
\hline RHP & $\checkmark$ & $x$ & $x$ & $x$ & $x$ \\
\hline LEP & $\checkmark$ & $\checkmark$ & $\checkmark$ & $\checkmark$ & $\checkmark$ \\
\hline LHP & $x$ & $\checkmark$ & $\checkmark$ & $\checkmark$ & $x$ \\
\hline MP & $x$ & $x$ & $\checkmark$ & $\checkmark$ & $\checkmark$ \\
\hline LKP & $\checkmark$ & $\checkmark$ & $x$ & $x$ & $\checkmark$ \\
\hline RKP & $\checkmark$ & $\checkmark$ & $\checkmark$ & $\checkmark$ & $x$ \\
\hline LFP & $\checkmark$ & $\checkmark$ & $\checkmark$ & $\checkmark$ & $\checkmark$ \\
\hline RFP & $\checkmark$ & $\checkmark$ & $\checkmark$ & $\checkmark$ & $\checkmark$ \\
\hline Accuracy & $81.81 \%$ & $72.72 \%$ & $72.72 \%$ & $72.72 \%$ & $72.72 \%$ \\
\hline \multicolumn{6}{|c|}{ Mean accuracy $=74.54 \%$} \\
\hline
\end{tabular}

1 


\section{Table 8(on next page)}

: Human body parts results of multi-person for mpii-video-pose dataset 


\begin{tabular}{|c|c|c|c|c|c|}
\hline Body parts & Human1 & Human2 & Human3 & Human4 & Human5 \\
\hline $\mathrm{HP}$ & $\checkmark$ & $\checkmark$ & $\checkmark$ & $\checkmark$ & $\checkmark$ \\
\hline NP & $x$ & $x$ & $\checkmark$ & $x$ & $\checkmark$ \\
\hline REP & $\checkmark$ & $\checkmark$ & $x$ & $\checkmark$ & $\checkmark$ \\
\hline RHP & $x$ & $x$ & $\checkmark$ & $x$ & $x$ \\
\hline LEP & $\checkmark$ & $\checkmark$ & $x$ & $\checkmark$ & $\checkmark$ \\
\hline LHP & $\checkmark$ & $x$ & $\checkmark$ & $x$ & $x$ \\
\hline MP & $x$ & $\checkmark$ & $\checkmark$ & $\checkmark$ & $\checkmark$ \\
\hline LKP & $\checkmark$ & $\checkmark$ & $x$ & $\checkmark$ & $\checkmark$ \\
\hline RKP & $\checkmark$ & $x$ & $\checkmark$ & $x$ & $x$ \\
\hline LFP & $\checkmark$ & $\checkmark$ & $x$ & $\checkmark$ & $\checkmark$ \\
\hline RFP & $x$ & $\checkmark$ & $\checkmark$ & $\checkmark$ & $\checkmark$ \\
\hline Accuracy & $63.63 \%$ & $63.63 \%$ & $63.63 \%$ & $63.63 \%$ & $72.72 .1 \%$ \\
\hline \multicolumn{6}{|c|}{ Mean accuracy $=65.45 \%$} \\
\hline
\end{tabular}

1 


\section{Table 9 (on next page)}

Precision, recall, and F-1 measure comparison with the artificial neural network, decision tree and CNN over Mpii-video-pose dataset 


\begin{tabular}{|c|c|c|c|c|c|c|c|c|c|}
\hline Event & \multicolumn{3}{|c|}{ Artificial Neural Network } & \multicolumn{3}{|c|}{ Decision Tree } & \multicolumn{3}{|c|}{ CNN } \\
\hline Events & $\begin{array}{c}\text { Precisi } \\
\text { on }\end{array}$ & Recall & $\begin{array}{c}\text { F- } 1 \\
\text { measure }\end{array}$ & Precision & Recall & $\begin{array}{c}\mathrm{F}-1 \\
\text { measure }\end{array}$ & Precision & Recall & $\begin{array}{c}\text { F- } 1 \\
\text { Measure }\end{array}$ \\
\hline $\mathrm{Bi}$ & 0.778 & 0.700 & 0.737 & 0.667 & 0.600 & 0.632 & 0.818 & 0.900 & 0.857 \\
\hline $\mathrm{Ce}$ & 0.700 & 0.700 & 0.700 & 0.700 & 0.700 & 0.700 & 0.692 & 0.900 & 0.783 \\
\hline $\mathrm{Da}$ & 0.857 & 0.600 & 0.706 & 0.818 & 0.900 & 0.857 & 0.909 & 0.909 & 0.909 \\
\hline $\mathrm{Fh}$ & 0.909 & 1.000 & 0.952 & 0.727 & 0.800 & 0.762 & 1.000 & 0.900 & 0.947 \\
\hline MP & 0.900 & 0.900 & 0.900 & 0.727 & 0.800 & 0.762 & 0.900 & 0.900 & 0.900 \\
\hline $\mathrm{Ra}$ & 0.889 & 0.800 & 0.842 & 0.889 & 0.800 & 0.842 & 1.000 & 0.900 & 0.947 \\
\hline SP & 0.727 & 0.889 & 0.800 & 0.833 & 1.000 & 0.909 & 0.900 & 0.900 & 0.900 \\
\hline $\operatorname{Tr}$ & 0.875 & 0.778 & 0.824 & 0.909 & 1.000 & 0.952 & 1.000 & 0.800 & 0.889 \\
\hline $\mathrm{Wl}$ & 0.875 & 0.700 & 0.778 & 1.000 & 0.700 & 0.824 & 1.000 & 0.818 & 0.900 \\
\hline $\mathrm{Wa}$ & 0.818 & 1.000 & 0.900 & 1.000 & 0.900 & 0.947 & 1.000 & 1.000 & 1.000 \\
\hline $\mathrm{Wn}$ & 0.769 & 1.000 & 0.870 & 0.800 & 0.800 & 0.800 & 0.750 & 0.900 & 0.818 \\
\hline Mean & 0.827 & 0.824 & 0.819 & 0.825 & 0.818 & 0.817 & 0.906 & 0.893 & 0.896 \\
\hline
\end{tabular}




\section{Table $\mathbf{1 0}$ (on next page)}

Precision, recall, and F-1 measure comparison with the artificial neural network, decision tree and CNN over COCO dataset. 


\begin{tabular}{|c|c|c|c|c|c|c|c|c|c|}
\hline Event & \multicolumn{3}{|c|}{ Artificial Neural Network } & \multicolumn{3}{|c|}{ Decision Tree } & \multicolumn{3}{|c|}{ CNN } \\
\hline Events & $\begin{array}{l}\text { Precisi } \\
\text { on }\end{array}$ & Recall & $\begin{array}{c}\text { F- } 1 \\
\text { measure }\end{array}$ & Precision & Recall & $\begin{array}{c}\mathrm{F}-1 \\
\text { measure }\end{array}$ & Precision & Recall & $\begin{array}{c}\text { F- } 1 \\
\text { Measure }\end{array}$ \\
\hline $\mathrm{Bi}$ & 0.818 & 0.750 & 0.783 & 0.700 & 0.700 & 0.700 & 0.889 & 0.800 & 0.842 \\
\hline $\mathrm{Da}$ & 0.750 & 0.750 & 0.750 & 0.889 & 0.800 & 0.842 & 0.909 & 1.000 & 0.952 \\
\hline $\mathrm{Ce}$ & 0.889 & 0.667 & 0.762 & 0.833 & 1.000 & 0.909 & 0.818 & 0.900 & 0.857 \\
\hline $\mathrm{Fh}$ & 0.900 & 1.000 & 0.947 & 0.818 & 0.900 & 0.857 & 1.000 & 0.800 & 0.889 \\
\hline $\mathrm{Ra}$ & 0.909 & 0.909 & 0.909 & 0.818 & 0.900 & 0.857 & 0.714 & 1.000 & 0.833 \\
\hline $\mathrm{Mp}$ & 0.900 & 0.818 & 0.857 & 0.900 & 0.900 & 0.900 & 0.889 & 0.800 & 0.842 \\
\hline $\mathrm{Sp}$ & 0.667 & 0.857 & 0.750 & 0.889 & 0.800 & 0.842 & 1.000 & 0.900 & 0.947 \\
\hline Wl & 0.900 & 0.818 & 0.857 & 0.727 & 1.000 & 0.842 & 1.000 & 0.900 & 0.947 \\
\hline $\operatorname{Tr}$ & 0.900 & 0.750 & 0.818 & 0.889 & 0.800 & 0.842 & 0.800 & 0.800 & 0.800 \\
\hline $\mathrm{Wa}$ & 0.800 & 1.000 & 0.889 & 1.000 & 0.800 & 0.889 & 1.000 & 0.900 & 0.947 \\
\hline Wn & 0.727 & 1.000 & 0.842 & 0.875 & 0.700 & 0.778 & 0.909 & 1.000 & 0.952 \\
\hline Mean & 0.833 & 0.847 & 0.833 & 0.849 & 0.845 & 0.842 & 0.903 & 0.891 & 0.892 \\
\hline
\end{tabular}




\section{Table $\mathbf{1 1}$ (on next page)}

Precision, recall, and F-1 measure comparison with the artificial neural network, decision tree and CNN over Posetrack dataset. 


\begin{tabular}{|c|c|c|c|c|c|c|c|c|c|}
\hline Event & \multicolumn{3}{|c|}{ Artificial Neural Network } & \multicolumn{3}{|c|}{ Decision Tree } & \multicolumn{3}{|c|}{ CNN } \\
\hline Events & $\begin{array}{c}\text { Precisi } \\
\text { on }\end{array}$ & Recall & $\begin{array}{c}\text { F- } 1 \\
\text { measure }\end{array}$ & Precision & Recall & $\begin{array}{c}\text { F- } 1 \\
\text { measure }\end{array}$ & Precision & Recall & $\begin{array}{c}\text { F- } 1 \\
\text { Measure }\end{array}$ \\
\hline $\mathrm{Ce}$ & 0.818 & 0.750 & 0.783 & 0.769 & 1.000 & 0.870 & 1.000 & 0.700 & 0.824 \\
\hline $\mathrm{Bi}$ & 0.700 & 0.700 & 0.700 & 0.750 & 0.818 & 0.783 & 1.000 & 0.800 & 0.889 \\
\hline $\mathrm{Da}$ & 0.889 & 0.667 & 0.762 & 0.875 & 0.700 & 0.778 & 0.900 & 0.900 & 0.900 \\
\hline $\mathrm{Mp}$ & 0.900 & 1.000 & 0.947 & 0.778 & 0.700 & 0.737 & 0.833 & 1.000 & 0.909 \\
\hline $\mathrm{Fh}$ & 0.875 & 0.875 & 0.875 & 0.692 & 0.900 & 0.783 & 0.769 & 1.000 & 0.870 \\
\hline $\mathrm{Ra}$ & 0.900 & 0.818 & 0.857 & 0.818 & 0.900 & 0.857 & 0.909 & 1.000 & 0.952 \\
\hline $\operatorname{Tr}$ & 0.750 & 0.900 & 0.818 & 0.700 & 0.700 & 0.700 & 0.889 & 0.800 & 0.842 \\
\hline $\mathrm{Sp}$ & 0.857 & 0.750 & 0.800 & 0.875 & 0.700 & 0.778 & 1.000 & 0.900 & 0.947 \\
\hline Wl & 0.857 & 0.667 & 0.750 & 1.000 & 0.750 & 0.857 & 0.833 & 1.000 & 0.909 \\
\hline Wn & 0.800 & 1.000 & 0.889 & 1.000 & 1.000 & 1.000 & 0.727 & 0.800 & 0.762 \\
\hline $\mathrm{Wa}$ & 0.769 & 1.000 & 0.870 & 0.778 & 0.778 & 0.778 & 1.000 & 0.800 & 0.889 \\
\hline Mean & 0.829 & 0.830 & 0.823 & 0.821 & 0.813 & 0.811 & 0.896 & 0.882 & 0.881 \\
\hline
\end{tabular}




\section{Table 12 (on next page)}

Performance analysis over various features extracting upon the MPII, COCO and Pose track datasets. 


\begin{tabular}{lccc}
\hline $\begin{array}{l}\text { Table9. Performance analysis over various features extracting upon the MPII, COCO and Pose track datasets. } \\
\text { Features combination }\end{array}$ & MPII (\%) & COCO (\%) & Pose Track (\%) \\
\hline DOF, $P M, N P M$ & 71.20 & 73.21 & 72.59 \\
$D O F, P M, N P M, M D F$ & 76.73 & 76.07 & 75.13 \\
DOF, PM, NPM,MDF, RAJ & 90.90 & 89.09 & 88.18 \\
\hline
\end{tabular}

1 


\section{Table $\mathbf{1 3}$ (on next page)}

Gait event mean accuracy comparison with the other methods over the MPII, COCO and Pose track datasets. 


\begin{tabular}{|c|c|c|c|c|c|}
\hline Methods & MPII (\%) & Methods & $\operatorname{COCO}(\%)$ & Methods & Pose Track (\%) \\
\hline Fan et al. (2015) & 73.00 & Sun et al. (2018) & 74.20 & Jin et al. (2019) & 71.08 \\
\hline $\begin{array}{c}\text { Pishchulin et al. } \\
\text { (2016) }\end{array}$ & 87.10 & $\begin{array}{c}\text { Rachmadi, } \\
\text { Uchimura \& } \\
\text { Koutaki (2016) }\end{array}$ & 82.30 & Bao et al. (2020) & 72.03 \\
\hline Wei et al. (2016) & 90.50 & Zhu et al.(2019) & 83.10 & $\begin{array}{l}\text { Umer et al. } \\
\text { (2020) }\end{array}$ & 74.02 \\
\hline $\begin{array}{l}\text { Proposed } \\
\text { method }\end{array}$ & 90.90 & & 89.09 & & 88.18 \\
\hline
\end{tabular}




\section{Table 14(on next page)}

Human body parts recognition and detection accuracy using Openpose CNN 


\begin{tabular}{ccccccc}
\multicolumn{6}{c}{ Table 11: Human body parts recognition and detection accuracy using Openpose CNN } \\
$\begin{array}{c}\text { Body key } \\
\text { points }\end{array}$ & Distance & $\begin{array}{c}\text { MPII } \\
\mathbf{( \% )}\end{array}$ & Distance & COCO (\%) & Distance & $\begin{array}{c}\text { Posetrack } \\
\mathbf{( \% )}\end{array}$ \\
\hline HP & 12.5 & 86 & 10.1 & 84 & 11.1 & 83 \\
NP & 12.3 & 83 & 12.3 & 83 & 12.5 & 81 \\
REP & 10.6 & 81 & 12.5 & 82 & 12.9 & 80 \\
RHP & 13.1 & 88 & 14.1 & 86 & 10.1 & 85 \\
LEP & 12.3 & 84 & 10.6 & 80 & 10.9 & 82 \\
LHP & 14.0 & 78 & 10.8 & 83 & 11.6 & 77 \\
MP & 11.3 & 88 & 12.2 & 88 & 9.9 & 92 \\
LKP & 11.0 & 85 & 11.9 & 87 & 10.5 & 89 \\
RKP & 15.1 & 83 & 13.3 & 89 & 12.8 & 85 \\
LFP & 14.2 & 90 & 11.6 & 91 & 11.3 & 91 \\
RFP & 12.3 & 88 & 11.1 & 93 & 10.1 & 92 \\
Mean Accuracy Rate & $\mathbf{8 4 . 9 0}$ & & $\mathbf{8 6 . 0 0}$ & & $\mathbf{8 5 . 1 8}$ \\
\hline
\end{tabular}




\section{Table 15(on next page)}

Comparison table the Openpose CNN model with the proposed method 
Table 12: Comparison table the Openpose CNN model with the proposed method

\begin{tabular}{ccc} 
Dataset & Openpose CNN & Proposed method \\
\hline MPII (\%) & 84.90 & 87.09 \\
COCO (\%) & 86.00 & 87.36 \\
Posetrack (\%) & 85.18 & 87.72 \\
\hline
\end{tabular}

1 